Research Article

\title{
Impacts of Backlash on Nonlinear Dynamic Characteristic of Encased Differential Planetary Gear Train
}

\author{
Zengbao Zhu $\mathbb{D}^{1},{ }^{1}$ Longchao Cheng, ${ }^{1}$ Rui Xu, ${ }^{1}$ and Rupeng Zhu $\mathbb{D}^{2}$ \\ ${ }^{1}$ College of Mechanical Engineering, Anhui University of Science and Technology, Huainan 232001, China \\ ${ }^{2}$ College of Mechanical and Electrical Engineering, Nanjing University of Aeronautics and Astronautics, Nanjing 210016, China
}

Correspondence should be addressed to Zengbao Zhu; zhbzhu@aust.edu.cn

Received 27 November 2018; Accepted 28 April 2019; Published 27 May 2019

Academic Editor: Francesco Pellicano

Copyright (C) 2019 Zengbao Zhu et al. This is an open access article distributed under the Creative Commons Attribution License, which permits unrestricted use, distribution, and reproduction in any medium, provided the original work is properly cited.

A multifreedom tensional nonlinear dynamic equation of encased differential planetary gear train with multibacklash and timevarying mesh stiffness was developed in the present research. The nonlinear dynamic response was obtained by solving the formulated nonlinear dynamic equation, and the impacts of backlash on dynamic characteristics of the gear train were then analyzed by combining time process diagram, phase diagram, and Poincaré section. The results revealed that bilateral shock in meshing teeth was caused due to smaller backlash, thus causing dramatic changes in meshing force; hence, the gears were found to be in a chaotic state. Further, during stable motion state, no contact between intermeshing teeth with bigger backlash was noticed; thus, they were in a stable quasiperiodic motion state in the absence of teeth exciting force. Therefore, in order to avoid a bilateral shock in gears as well as to maintain gear teeth lubrication, a slightly bigger backlash is required. The backlash change in any transmission stage caused significant impacts on gear force and the motion state of its own stage; however, the impact on gear force of another stage was quite small, whereas the impact on the motion state of another stage was quite large.

\section{Introduction}

Gear error, time-varying mesh stiffness, and backlash are often observed during internal excitation of a gear train [1]. Generally, gear error and time-varying mesh stiffness belong to the parametric vibration problem of a linear system [2]; hence, the influence of backlash on gear train is strongly nonlinear [3-5], and thus the encased differential planetary gear train can be considered as a nonlinear system with multibacklash. The encased differential planetary gear train is widely used in aero engine, crane, and ship powertrains [6]. In an actual gear train, a catastrophic accident occurs due to abnormal vibration in the system; thus, its dynamic behavior cannot be effectively explained by linear vibration theory [7]; therefore, it is necessary to study the nonlinear dynamic characteristics of encased differential planetary gear train. Some researchers [8-11] have already accurately modeled the nonlinear dynamics of a pair of gear pairs; it expedites the future study on nonlinear dynamic behavior of a gear train.
Kahraman and Singh [12, 13] studied the nonlinear dynamic behavior of a pair of gear pairs using the numerical integration and harmonic balance method in order to observe subharmonic resonance and chaotic phenomena. Sun [14] examined the influence of backlash on dynamic characteristics of planetary gear train by the harmonic balance method and Runge-Kutta numerical method. Sun et al. [15] analyzed the dynamic characteristics of encased differential planetary gear train without considering backlash, and in turn the nonlinear dynamic characteristics caused by backlash were not reported.

Due to time-varying meshing stiffness, the stiffness matrix of nonlinear dynamic equations of encased differential planetary gear train is generally found as singular; hence, the Runge-Kutta numerical integration method cannot be used to solve those equations because it cannot properly solve singular or morbid matrix problems [16]. In our research, the Newmark direct integration method of unconditional convergence was used to solve the nonlinear 
dynamics of gear train because of its better convergence accuracy and calculation speed [17].

In the present paper, a multifreedom torsional nonlinear dynamic equation of encased differential planetary gear train with multibacklash and time-varying meshing stiffness was established. The differential equations for eliminating rigid body displacement were obtained by coordinate transformation, and these equations were treated as dimensionless. Furthermore, the impacts of backlash on dynamics characteristics of gear train were analyzed by time process diagram, phase diagram, and Poincaré section.

\section{Dynamic Model of Transmission System}

The encased differential planetary train consisted of a differential stage and an encased stage, and its transmission diagram is presented in Figure 1. The differential stage was composed of a sun gear $Z_{\mathrm{s} 1}$, a planetary gear $Z_{\mathrm{p} i}(i=1,2, \ldots$, $N$ ), an internal gear $Z_{\mathrm{r} 1}$, and a planet carrier $\mathrm{H}$, whereas the encased stage was comprised of a sun gear $Z_{\mathrm{s} 2}$, a planetary gear $Z_{\mathrm{m} j}(j=1,2, \ldots, M)$, and an internal gear $Z_{\mathrm{r} 2}$. The input torque $\left(T_{\mathrm{D}}\right)$ was first shunted into the planet carrier $\mathrm{H}$ and the internal gear $Z_{\mathrm{r} 1}$ through the differential stage sun gear $Z_{\mathrm{s} 1}$ and then converged to the output shaft (L).

The dynamic model of encased differential planetary gear train system is displayed in Figure 2. A moving coordinate system was designed for the differential stage, where the planet carrier rotated at $\omega_{\mathrm{H}}$ (Figure $2(\mathrm{a})$ ), whereas a fixed coordinate system was used in the encased stage (Figure 2(b)).

In Figure $2, K_{\mathrm{HL}}, K_{\mathrm{r} 1 \mathrm{~s} 2}$, and $K_{\mathrm{r} 2 \mathrm{~L}}$ are the torsional stiffness of coupling $\mathrm{H}$ and $\mathrm{L}, Z_{\mathrm{r} 1}$ and $Z_{\mathrm{s} 2}$, and $Z_{\mathrm{r} 2}$ and $\mathrm{L}$, respectively. $K_{\mathrm{s} 1}, K_{\mathrm{p}}, K_{\mathrm{r} 1}, K_{\mathrm{s} 2}, K_{\mathrm{m}}$, and $K_{\mathrm{r} 2}$ are the support bending rigidities of gears $Z_{\mathrm{s} 1}, Z_{\mathrm{p} i}, Z_{\mathrm{r} 1}, Z_{\mathrm{s} 2}, Z_{\mathrm{m} j}$, and $Z_{\mathrm{r} 2}$, respectively. $K_{\mathrm{H}}$ denotes the equivalent tangential stiffness of planet carrier $\mathrm{H}$ on the revolution radius $\left(r_{\mathrm{H}}\right)$ of planetary gear $Z_{\mathrm{p} i}$.

The encased differential planetary train possessed a total of $(6+N+M)$ degree of freedom, which was represented by the torsion line displacement column vector $\mathbf{X}$.

$$
\mathbf{X}=\left\{x_{\mathrm{s} 1}, x_{\mathrm{p} 1}, x_{\mathrm{r} 1}, x_{\mathrm{s} 2}, x_{\mathrm{m} j}, x_{\mathrm{r} 2}, x_{\mathrm{H}}, x_{\mathrm{L}}\right\}^{\mathrm{T}},
$$

where $x_{\mathrm{s} 1}, x_{\mathrm{p} i}, x_{\mathrm{r} 1}, x_{\mathrm{s} 2}, x_{\mathrm{m} j}$, and $x_{\mathrm{r} 2}$ are the line displacements of gears $Z_{\mathrm{s} 1}, Z_{\mathrm{p} i}, Z_{\mathrm{r} 1}, Z_{\mathrm{s} 2}, Z_{\mathrm{m} j}$, and $Z_{\mathrm{r} 2}$, respectively, along the base circle, $x_{\mathrm{H}}$ signifies the line displacement of planet carrier $\mathrm{H}$ along its base circle radius $r_{\mathrm{H}}$, and $x_{\mathrm{L}}$ denotes the line displacement at the contact of output shaft $\mathrm{L}$ and gear $Z_{\text {r2 }}$.

\section{Dynamic Equilibrium Equation of Transmission System}

3.1. Elastic Meshing Force of Gear Pair. The internal and external elastic meshing forces, $F_{\mathrm{rp} i}(t)$ and $F_{\mathrm{spi}}(t)$, of differential stage gear pairs were expressed by the following equation:

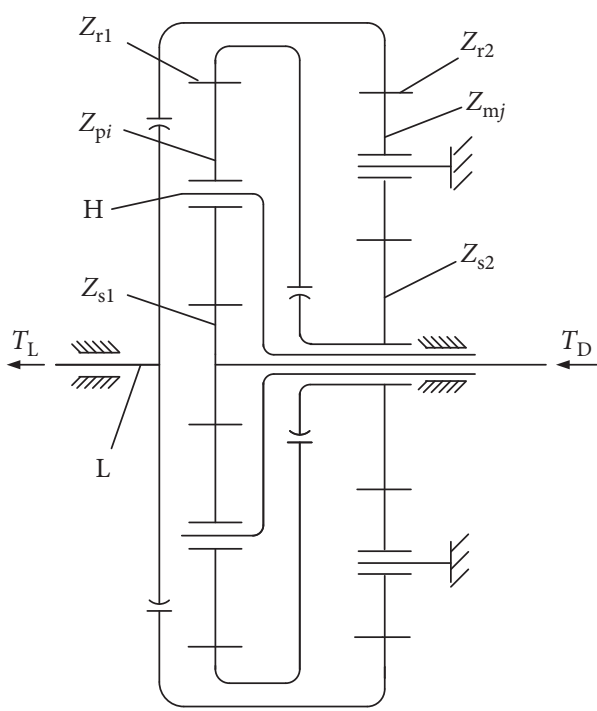

FIGURE 1: Schematic diagram of encased differential planetary gear train.

$$
\left\{\begin{array}{l}
F_{\mathrm{rp} i}(t)=K_{\mathrm{rp} i}(t) f\left(X_{\mathrm{rp} i}, b_{\mathrm{rp} i}\right), \\
F_{\mathrm{sp} i}(t)=K_{\mathrm{sp} i}(t) f\left(X_{\mathrm{sp} i}, b_{\mathrm{sp} i}\right) .
\end{array}\right.
$$

The internal and external elastic meshing forces, $F_{\mathrm{rm} j}(t)$ and $F_{\text {sm } j}(t)$, of encased stage gear pairs were formulated by the following equation:

$$
\left\{\begin{array}{l}
F_{\mathrm{rm} j}(t)=K_{\mathrm{rm} j}(t) f\left(X_{\mathrm{rm} j}, b_{\mathrm{rm} j}\right), \\
F_{\mathrm{sm} j}(t)=K_{\mathrm{sm} j}(t) f\left(X_{\mathrm{sm} j}, b_{\mathrm{sm} j}\right),
\end{array}\right.
$$

where $X_{\mathrm{rp} i}$ and $X_{\mathrm{sp} i}$ are the line displacements of internal and external meshing of differential stage, respectively, $X_{\mathrm{rm} j}$ and $X_{\mathrm{sm} j}$ are the line displacements of internal and external meshing of encased stage, respectively, $b_{\mathrm{rp} i}$ and $b_{\mathrm{sp} i}$ denote the backlashes of internal and external meshing of differential stage, respectively, $b_{\mathrm{rm} i}$ and $b_{\mathrm{sm} i}$ denote the backlashes of internal and external meshing of encased stage, respectively, $K_{\mathrm{rp} i}(t)$ and $K_{\mathrm{sp} i}(t)$ represent the time-varying mesh stiffness of internal and external meshing of differential stage, respectively, and $K_{\mathrm{rm} j}(t)$ and $K_{\mathrm{sm} j}(t)$ signify the time-varying mesh stiffness of internal and external meshing of encased stage, respectively. The time-varying meshing stiffness of spur gear was calculated according to the formula derived by Maatar and Velex [18], and the meshing phase difference was estimated according to the method proposed by Parker and Lin [19]. $f\left(X_{\mathrm{rp} i}, b_{\mathrm{rp} i}\right), f\left(X_{\mathrm{sp} i}, b_{\mathrm{sp} i}\right), f\left(X_{\mathrm{rm} j}, b_{\mathrm{rm} j}\right)$, and $f\left(X_{\mathrm{sm} j}, b_{\mathrm{sm} j}\right)$ are gap nonlinear functions.

$$
\begin{aligned}
& f\left(X_{k}, b_{k}\right)= \begin{cases}X_{k}-b_{k}, & x_{k}>b_{k}, \\
0, & -b_{k} \leq x_{k} \leq b_{k}, \\
X_{k}+b_{k}, & x_{k}<-b_{k},\end{cases} \\
& \quad(k=1,2, \ldots, 2 N, 2 N+1, \ldots, 2 N+2 M),
\end{aligned}
$$




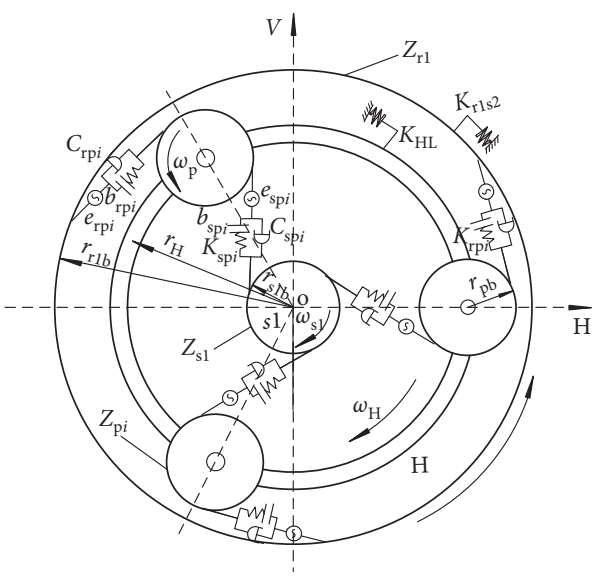

(a)

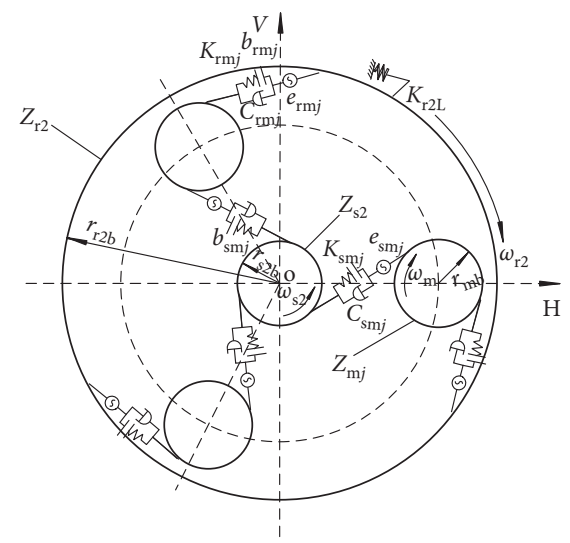

(b)

Figure 2: Dynamic model of encased differential planetary gear train. (a) Differential stage dynamic model. (b) Encased stage dynamic model.

where $k$ is the number of internal and external meshing lines of differential and encased stages and $X_{k}$ and $b_{k}$ denote mesh line displacement and backlash of each gear pair, respectively.

3.2. Meshing Damping Force of Gear Pair. The internal and external meshing damping forces, $D_{\mathrm{rpi}}(t)$ and $D_{\mathrm{sp} i}(t)$, of differential stage gear pairs were calculated by the following equation:

$$
\left\{\begin{array}{l}
D_{\mathrm{rp} i}(t)=C_{\mathrm{rp}} \dot{X}_{\mathrm{rp} i} \\
D_{\mathrm{spi}}(t)=C_{\mathrm{sp}} \dot{X}_{\mathrm{sp} i}
\end{array}\right.
$$

The internal and external meshing damping forces, $D_{\mathrm{rm} j}$ $(t)$ and $D_{\text {smj }}(t)$, of encased stage gear pairs were estimated by the following equation:

$$
\left\{\begin{array}{l}
D_{\mathrm{rm} j}(t)=C_{\mathrm{rm}} \dot{X}_{\mathrm{rm} j}, \\
D_{\mathrm{sm} j}(t)=C_{\mathrm{sm}} \dot{X}_{\mathrm{sm} j},
\end{array}\right.
$$

where $C_{\mathrm{rp}}, C_{\mathrm{sp}}, C_{\mathrm{rm}}$, and $C_{\mathrm{sm}}$ signify meshing damping coefficients of each gear pair.

3.3. Basic Equations of Dynamics. The dynamic equilibrium equations of pure torsion in encased differential planetary gear train were expressed by

$$
\left\{\begin{array}{l}
m_{\mathrm{s} 1} \ddot{x}_{\mathrm{s} 1}+\sum_{i=1}^{N}\left(F_{\mathrm{sp} i}(t)+D_{\mathrm{sp} i}(t)\right)=P_{\mathrm{D}} \\
m_{\mathrm{p}} \ddot{x}_{\mathrm{p} i}-\left(F_{\mathrm{sp} i}(t)+D_{\mathrm{sp} i}(t)\right)+\left(F_{\mathrm{rp} i}(t)+D_{\mathrm{rp} i}(t)\right)=0 \\
m_{\mathrm{r} 1} \ddot{x}_{\mathrm{r} 1}-\sum_{i=1}^{N}\left(F_{\mathrm{rp} i}(t)+D_{\mathrm{rp} i}(t)\right)+\frac{K_{\mathrm{r} 1 \mathrm{~s} 2}}{r_{\mathrm{r} 1 \mathrm{~b}}}\left(\frac{x_{\mathrm{r} 1}}{r_{\mathrm{r} 1 \mathrm{~b}}}-\frac{x_{\mathrm{s} 2}}{r_{\mathrm{s} 2 \mathrm{~b}}}\right)=0 \\
m_{\mathrm{s} 2} \ddot{x}_{\mathrm{s} 2}+\sum_{j=1}^{M}\left(F_{\mathrm{sm} j}(t)+D_{\mathrm{sm} j}(t)\right)-\frac{K_{\mathrm{r} 1 \mathrm{~s} 2}}{r_{\mathrm{s} 2 \mathrm{~b}}}\left(\frac{x_{\mathrm{r} 1}}{r_{\mathrm{r} 1 \mathrm{~b}}}-\frac{x_{\mathrm{s} 2}}{r_{\mathrm{s} 2 \mathrm{~b}}}\right)=0 \\
m_{\mathrm{m}} \ddot{x}_{\mathrm{m} j}-\left(F_{\mathrm{sm} j}(t)+D_{\mathrm{sm} j}(t)\right)+\left(F_{\mathrm{rm} j}(t)+D_{\mathrm{rm} j}(t)\right)=0 \\
m_{\mathrm{r} 2} \ddot{x}_{\mathrm{r} 2}-\sum_{j=1}^{M}\left(F_{\mathrm{rm} j}(t)+D_{\mathrm{rm} j}(t)\right)+\frac{K_{\mathrm{r} 2 \mathrm{~L}}}{r_{\mathrm{r} 2 \mathrm{~b}}}\left(\frac{x_{\mathrm{r} 2}}{r_{\mathrm{r} 2 \mathrm{~b}}}-\frac{x_{\mathrm{L}}}{r_{\mathrm{L}}}\right)=0 \\
m_{\mathrm{L}} \ddot{x}_{\mathrm{L}}-\frac{K_{\mathrm{HL}}}{r_{\mathrm{L}}}\left(\frac{x_{\mathrm{H}}}{r_{\mathrm{H}}}-\frac{x_{\mathrm{L}}}{r_{\mathrm{L}}}\right)-\frac{k_{\mathrm{r} 2 \mathrm{~L}}}{r_{\mathrm{r} 2 \mathrm{~b}}}\left(\frac{x_{\mathrm{r} 2}}{\left.r_{\mathrm{r} 2 \mathrm{~b}}-\frac{x_{\mathrm{L}}}{r_{\mathrm{L}}}\right)=-P_{\mathrm{L}}}\right. \\
\left.+\frac{K_{\mathrm{HL}}}{r_{\mathrm{H}}}+N \frac{x_{\mathrm{H}}}{r_{\mathrm{H}}}-\frac{x_{\mathrm{L}}}{r_{\mathrm{L}}}\right)=0 \\
\left.m_{\mathrm{p}}\right) \ddot{x}_{\mathrm{H}}-\sum_{i=1}^{N}\left(F_{\mathrm{sp} i}(t)+D_{\mathrm{sp} i}(t)+F_{\mathrm{rp} i}(t)+D_{\mathrm{rp} i}(t)\right)
\end{array}\right.
$$

where $m_{\mathrm{s} 1}, m_{\mathrm{p}}, m_{\mathrm{r} 1}, m_{\mathrm{s} 2}, m_{\mathrm{m}}$, and $m_{\mathrm{r} 2}$ are the equivalent masses of sun gear $Z_{\mathrm{s} 1}$, planetary gear $Z_{\mathrm{p} i}$, internal gear $Z_{\mathrm{r} 1}$, sun gear $Z_{\mathrm{s} 2}$, planetary gear $Z_{\mathrm{m} j}$, and internal gear $Z_{\mathrm{r} 2}$ on their respective base circle radii, $m_{\mathrm{H}}$ is the equivalent mass of planet carrier $\mathrm{H}$ on the revolution radius $\left(r_{\mathrm{H}}\right)$ of planetary gear, and $m_{\mathrm{L}}$ denotes the equivalent mass of output shaft $\mathrm{L}$ at the contact between output shaft $\mathrm{L}$ and gear $Z_{\mathrm{r} 2} . P_{\mathrm{D}}$ is the equivalent force of input torque $T_{\mathrm{D}}$ on the base circle radius $\left(r_{\mathrm{s} 1 \mathrm{~b}}\right)$ of sun gear $Z_{\mathrm{s} 1}$; therefore, $P_{\mathrm{D}}=T_{\mathrm{D}} / r_{\mathrm{s} 1 \mathrm{~b}} . P_{\mathrm{L}}$ is the equivalent force of load torque $T_{\mathrm{L}}$ at the contact between output shaft $\mathrm{L}$ and internal gear $Z_{\mathrm{r} 2}$; hence, $P_{\mathrm{L}}=T_{\mathrm{L}} / r_{\mathrm{r} 2 \mathrm{~b}}$, where $r_{\mathrm{r} 2 \mathrm{~b}}$ is the base circle radius of internal gear $Z_{\mathrm{r} 2}$.

3.4. Coordinate Transformation of Kinetic Equations. In order to facilitate the calculation of equation (7), relative displacements between adjacent components were introduced. The coordinate transformation of equation (7) was formulated according to the following equations: 


$$
\begin{aligned}
& \left\{\begin{array}{l}
X_{\mathrm{sp} i}=x_{\mathrm{s} 1}-x_{\mathrm{p} i}-e_{\mathrm{sp} i}(t), \\
X_{\mathrm{s} 1 \mathrm{r} 1}=x_{\mathrm{s} 1}-x_{\mathrm{r} 1}, \\
X_{\mathrm{r} 1 \mathrm{~s} 2}=x_{\mathrm{r} 1}-\left(\frac{r_{\mathrm{r} 1 \mathrm{~b}}}{r_{\mathrm{s} 2 \mathrm{~b}}}\right) x_{\mathrm{s} 2}, \\
X_{\mathrm{sm} j}=x_{\mathrm{s} 2}-x_{\mathrm{m} j}-e_{\mathrm{sm} j}(t), \\
X_{\mathrm{s} 2 \mathrm{r} 2}=x_{\mathrm{s} 2}-x_{\mathrm{r} 2}, \\
X_{\mathrm{r} 2 \mathrm{~L}}=x_{\mathrm{r} 2}-\left(\frac{r_{\mathrm{r} 2 \mathrm{~b}}}{r_{\mathrm{L}}}\right) x_{\mathrm{L}}, \\
X_{\mathrm{HL}}=x_{\mathrm{H}}-\left(\frac{r_{\mathrm{H}}}{r_{\mathrm{L}}}\right) x_{\mathrm{L}} .
\end{array}\right. \\
& \left\{\begin{array}{l}
X_{\mathrm{rp} i}=x_{\mathrm{p} i}-x_{\mathrm{r} 1}-e_{\mathrm{rp} i}(t), \\
X_{\mathrm{rm} j}=x_{\mathrm{m} j}-x_{\mathrm{r} 2}-e_{\mathrm{rm} j}(t) .
\end{array}\right.
\end{aligned}
$$

Therefore,

$$
\left\{\begin{array}{l}
X_{\mathrm{rp} i}=X_{\mathrm{s} 1 \mathrm{r} 1}-X_{\mathrm{sp} i}-e_{\mathrm{sp} i}(t)-e_{\mathrm{rp} i}(t), \\
X_{\mathrm{rm} j}=X_{\mathrm{s} 2 \mathrm{r} 2}-X_{\mathrm{sm} j}-e_{\mathrm{sm} j}(t)-e_{\mathrm{rm} j}(t),
\end{array}\right.
$$

where $e_{\mathrm{rp} i}(t), e_{\mathrm{sp} i}(t), e_{\mathrm{rm} j}(t)$, and $e_{\mathrm{sm} j}(t)$ are the comprehensive error equivalent displacements between $Z_{\mathrm{p} i}$ and $Z_{\mathrm{r} 1}$,
$Z_{\mathrm{s} 1}$ and $Z_{\mathrm{p} i}, Z_{\mathrm{m} j}$ and $Z_{\mathrm{r} 2}$, and $Z_{\mathrm{s} 2}$ and $Z_{\mathrm{m} j}$ in equations (8)-(10), respectively.

The equivalent masses, $M_{\mathrm{sp}}, M_{\mathrm{s} 1 \mathrm{r} 1}, M_{\mathrm{r} 1 \mathrm{~s} 2}, M_{\mathrm{sm}}, M_{\mathrm{s} 2 \mathrm{r} 2}$, $M_{\mathrm{r} 2 \mathrm{~L}}$, and $M_{\mathrm{HL}}$, were expressed by

$$
\left\{\begin{array}{l}
M_{\mathrm{sp}}=\frac{m_{\mathrm{s} 1} m_{\mathrm{p}}}{m_{\mathrm{s} 1}+m_{\mathrm{p}}}, \\
M_{\mathrm{s} 1 \mathrm{r} 1}=\frac{m_{\mathrm{s} 1} m_{\mathrm{r} 1}}{m_{\mathrm{s} 1}+m_{\mathrm{r} 1}} \\
M_{\mathrm{r} 1 \mathrm{~s} 2}=\frac{m_{\mathrm{r} 1} m_{\mathrm{s} 2}}{m_{\mathrm{r} 1}+m_{\mathrm{s} 2}} \\
M_{\mathrm{sm}}=\frac{m_{\mathrm{s} 2} m_{\mathrm{m}}}{m_{\mathrm{s} 2}+m_{\mathrm{m}}}, \\
M_{\mathrm{s} 2 \mathrm{r} 2}=\frac{m_{\mathrm{s} 2} m_{\mathrm{r} 2}}{m_{\mathrm{s} 2}+m_{\mathrm{r} 2}} \\
M_{\mathrm{r} 2 \mathrm{~L}}=\frac{m_{\mathrm{r} 2} m_{\mathrm{L}}}{m_{\mathrm{r} 2}+m_{\mathrm{L}}}, \\
M_{\mathrm{HL}}=\frac{m_{\mathrm{H}} m_{\mathrm{L}}}{m_{\mathrm{H}}+m_{\mathrm{L}}} .
\end{array}\right.
$$

Now, by incorporating equation (8) into equation (11), the coordinate transformation of equation (7) was expressed by the following equations:

$$
\begin{aligned}
& M_{\mathrm{sp}} \ddot{X}_{\mathrm{sp} i}+\frac{M_{\mathrm{sp}}}{m_{\mathrm{s} 1}} \sum_{i=1}^{N}\left(K_{\mathrm{sp} i}(t) f\left(X_{\mathrm{sp} i}, b_{\mathrm{sp} i}\right)+C_{\mathrm{sp}} \dot{X}_{\mathrm{sp} i}\right)+\frac{M_{\mathrm{sp}}}{m_{\mathrm{p}}}\left(K_{\mathrm{sp} i}(t) f\left(X_{\mathrm{sp} i}, b_{\mathrm{sp} i}\right)+C_{\mathrm{sp}} \dot{X}_{\mathrm{sp} i}\right) \\
& -\frac{M_{\mathrm{sp}}}{m_{\mathrm{p}}}\left(K_{\mathrm{rp} i}(t) f\left(X_{\mathrm{s} 1 \mathrm{r} 1}-X_{\mathrm{sp} i}-e_{\mathrm{sp} i}(t)-e_{\mathrm{rp} i}(t), b_{\mathrm{rp} i}\right)\right)+C_{\mathrm{rp}}\left(\dot{X}_{\mathrm{s} 1 \mathrm{r} 1}-\dot{X}_{\mathrm{sp} i}-\dot{e}_{\mathrm{sp} i}(t)-\dot{e}_{\mathrm{rp} i}(t)\right)=\frac{M_{\mathrm{sp}}}{m_{\mathrm{s} 1}} P_{\mathrm{D}}-M_{\mathrm{sp}} \ddot{e}_{\mathrm{sp} i}(t),
\end{aligned}
$$

$$
\begin{aligned}
& M_{\mathrm{s} 1 \mathrm{r} 1} \ddot{X}_{\mathrm{s} 1 \mathrm{r} 1}+\frac{M_{\mathrm{s} 1 \mathrm{r} 1}}{m_{\mathrm{s} 1}} \sum_{i=1}^{N}\left(K_{\mathrm{sp} i}(t) f\left(X_{\mathrm{sp} i}, b_{\mathrm{sp} i}\right)+C_{\mathrm{sp}} X_{\mathrm{sp} i}\right)+\frac{M_{\mathrm{s} 1 \mathrm{r} 1}}{m_{\mathrm{r} 1}} \sum_{i=1}^{N}\left(K_{\mathrm{rp} i}(t) f\left(X_{\mathrm{s} 1 \mathrm{r} 1}-X_{\mathrm{sp} i}-e_{\mathrm{sp} i}(t)-e_{\mathrm{rp} i}(t), b_{\mathrm{rp} i}\right)\right. \\
& \quad+C_{\mathrm{rp}}\left(\dot{X}_{\mathrm{s} 1 \mathrm{r} 1}-\dot{X}_{\mathrm{sp} i}-\dot{e}_{\mathrm{sp} i}(t)-e_{\mathrm{rpi}}(t)\right)-\frac{M_{\mathrm{s} 1 \mathrm{r} 1} K_{\mathrm{r} 1 \mathrm{~s} 2}}{m_{\mathrm{r} 1} r_{\mathrm{rlb}}^{2}} X_{\mathrm{r} 1 \mathrm{~s} 2}=\frac{M_{\mathrm{slr} 1}}{m_{\mathrm{s} 1}} P_{\mathrm{D}},
\end{aligned}
$$

$$
\begin{aligned}
& M_{\mathrm{r} 1 \mathrm{~s} 2} \ddot{X}_{\mathrm{r} 1 \mathrm{~s} 2}-\frac{M_{\mathrm{r} 1 \mathrm{~s} 2}}{m_{\mathrm{r} 1}} \sum_{i=1}^{N}\left(K_{\mathrm{rp} i}(t) f\left(X_{\mathrm{s} 1 \mathrm{r} 1}-X_{\mathrm{sp} i}-e_{\mathrm{sp} i}(t)-e_{\mathrm{rp} i}(t), b_{\mathrm{rp} i}\right)+C_{\mathrm{rp}}\left(\dot{X}_{\mathrm{s} 1 \mathrm{r} 1}-\dot{X}_{\mathrm{sp} i}-\dot{e}_{\mathrm{sp} i}(t)-\dot{e}_{\mathrm{rp} i}(t)\right)\right) \\
& +\frac{M_{\mathrm{r} 1 \mathrm{~s} 2} K_{\mathrm{r} 1 \mathrm{~s} 2}}{m_{\mathrm{r} 1} r_{\mathrm{r} 1 \mathrm{~b}}^{2}} X_{\mathrm{r} 1 \mathrm{~s} 2}-\frac{r_{\mathrm{r} 1 \mathrm{~b}}}{r_{\mathrm{s} 2 \mathrm{~b}}} \frac{M_{\mathrm{r} 1 \mathrm{~s} 2}}{m_{\mathrm{s} 2}} \sum_{j=1}^{M}\left(K_{\mathrm{sm} j}(t) f\left(X_{\mathrm{sm} j}, b_{\mathrm{sm} j}\right)+C_{\mathrm{sm}} \dot{X}_{\mathrm{sm} j}\right)+\frac{M_{\mathrm{r} 1 \mathrm{~s} 2} K_{\mathrm{r} 1 \mathrm{~s} 2}}{m_{\mathrm{s} 2} r_{\mathrm{s} 2 \mathrm{~b}}^{2}} X_{\mathrm{r} 1 \mathrm{~s} 2}=0,
\end{aligned}
$$




$$
\begin{aligned}
& M_{\mathrm{sm}} \ddot{X}_{\mathrm{sm} j}+\frac{M_{\mathrm{sm}}}{m_{\mathrm{s} 2}} \sum_{j=1}^{M}\left(K_{\mathrm{sm} j}(t) f\left(X_{\mathrm{sm} j}, b_{\mathrm{sm} j}\right)+C_{\mathrm{sm}} \dot{X}_{\mathrm{sm} j}\right)-\frac{M_{\mathrm{sm}} K_{\mathrm{r} 1 \mathrm{~s} 2}}{m_{\mathrm{s} 2} r_{\mathrm{r} 1 \mathrm{~b}} r_{\mathrm{s} 2 \mathrm{~b}}} X_{\mathrm{r} 1 \mathrm{~s} 2}+\frac{M_{\mathrm{sm}}}{m_{\mathrm{m}}}\left(K_{\mathrm{sm} j}(t) f\left(X_{\mathrm{sm} j}, b_{\mathrm{sm} j}\right)+C_{\mathrm{sm}} \dot{X}_{\mathrm{sm} j}\right) \\
& -\frac{M_{\mathrm{sm}}}{m_{\mathrm{m}}}\left(K_{\mathrm{rm} j}(t) f\left(X_{\mathrm{s} 2 \mathrm{r} 2}-X_{\mathrm{sm} j}-e_{\mathrm{sm} j}(t)-e_{\mathrm{rm} j}(t), b_{\mathrm{rm} j}\right)+C_{\mathrm{rm}}\left(\dot{X}_{\mathrm{s} 2 \mathrm{r} 2}-\dot{X}_{\mathrm{sm} j}-\dot{e}_{\mathrm{sm} j}(t)-\dot{e}_{\mathrm{rm} j}(t)\right)\right)=-M_{\mathrm{sm}} \ddot{e}_{\mathrm{sm} j}(t), \\
& M_{\mathrm{s} 2 \mathrm{r} 2} \ddot{X}_{\mathrm{s} 2 \mathrm{r} 2}+\frac{M_{\mathrm{s} 2 \mathrm{r} 2}}{m_{\mathrm{s} 2}} \sum_{j=1}^{M}\left(K_{\mathrm{sm} j}(t) f\left(X_{\mathrm{sm} j}, b_{\mathrm{sm} j}\right)+C_{\mathrm{sm}} \dot{X}_{\mathrm{sm} j}\right)-\frac{M_{\mathrm{s} 2 \mathrm{r} 2} K_{\mathrm{r} 1 \mathrm{~s} 2}}{m_{\mathrm{s} 2} r_{\mathrm{r} 1 \mathrm{~b}} r_{\mathrm{s} 2 \mathrm{~b}}} X_{\mathrm{r} 1 \mathrm{~s} 2} \\
& +\frac{M_{\mathrm{s} 2 \mathrm{r} 2}}{m_{\mathrm{r} 2}} \sum_{j=1}^{M}\left(K_{\mathrm{rm} j}(t) f\left(X_{\mathrm{s} 2 \mathrm{r} 2}-X_{\mathrm{sm} j}-e_{\mathrm{sm} j}(t)-e_{\mathrm{rm} j}(t), b_{\mathrm{rm} j}\right)+C_{\mathrm{rm}}\left(\dot{X}_{\mathrm{s} 2 \mathrm{r} 2}-\dot{X}_{\mathrm{sm} j}-\dot{e}_{\mathrm{sm} j}(t)-\dot{e}_{\mathrm{rm} j}(t)\right)\right) \\
& -\frac{M_{\mathrm{s} 2 \mathrm{r} 2} K_{\mathrm{r} 2 \mathrm{~L}}}{m_{\mathrm{r} 2} r_{\mathrm{r} 2 \mathrm{~b}}^{2}} X_{\mathrm{r} 2 \mathrm{~L}}=0 \\
& M_{\mathrm{r} 2 \mathrm{~L}} \ddot{X}_{\mathrm{r} 2 \mathrm{~L}}-\frac{M_{\mathrm{r} 2 \mathrm{~L}}}{m_{\mathrm{r} 2}} \sum_{j=1}^{M}\left(K_{\mathrm{rm} j}(t) f\left(X_{\mathrm{s} 2 \mathrm{r} 2}-X_{\mathrm{sm} j}-e_{\mathrm{sm} j}(t)-e_{\mathrm{rm} j}(t), b_{\mathrm{rm} j}\right)+C_{\mathrm{rm}}\left(\dot{X}_{\mathrm{s} 2 \mathrm{r} 2}-\dot{X}_{\mathrm{sm} j}-\dot{e}_{\mathrm{sm} j}(t)-\dot{e}_{\mathrm{rm} j}(t)\right)\right) \\
& +\frac{M_{\mathrm{r} 2 \mathrm{~L}} K_{\mathrm{r} 2 \mathrm{~L}}}{m_{\mathrm{r} 2} r_{\mathrm{r} 2 \mathrm{~b}}^{2}} X_{\mathrm{r} 2 \mathrm{~L}}+\frac{M_{\mathrm{r} 2 \mathrm{~L}} r_{\mathrm{r} 2 \mathrm{~b}} K_{\mathrm{HL}}}{m_{\mathrm{L}} r_{\mathrm{H}} r_{\mathrm{L}}^{2}} X_{\mathrm{HL}}+\frac{M_{\mathrm{r} 2 \mathrm{~L}} k_{\mathrm{r} 2 \mathrm{~L}}}{r_{\mathrm{L}} m_{\mathrm{L}} r_{\mathrm{r} 2 \mathrm{~b}}} X_{\mathrm{r} 2 \mathrm{~L}}=\frac{M_{\mathrm{r} 2 \mathrm{~L}} r_{\mathrm{r} 2 \mathrm{~b}}}{r_{\mathrm{L}} m_{\mathrm{L}}} P_{\mathrm{L}} \\
& M_{\mathrm{HL}} \ddot{X}_{\mathrm{HL}}-\frac{M_{\mathrm{HL}}}{\left(m_{\mathrm{H}}+N m_{\mathrm{p}}\right)} \sum_{i=1}^{N}\left(K_{\mathrm{sp} i}(t) f\left(X_{\mathrm{sp} i}, b_{\mathrm{sp} i}\right)+C_{\mathrm{sp}} \dot{X}_{\mathrm{sp} i}+K_{\mathrm{rp} i}(t) f\left(X_{\mathrm{s} 1 \mathrm{r} 1}-X_{\mathrm{sp} i}-e_{\mathrm{sp} i}(t)-e_{\mathrm{rp} i}(t), b_{\mathrm{rp} i}\right)\right. \\
& \left.+C_{\mathrm{rp}}\left(\dot{X}_{\mathrm{s} 1 \mathrm{r} 1}-\dot{X}_{\mathrm{sp} i}-\dot{e}_{\mathrm{sp} i}(t)-\dot{e}_{\mathrm{rpi}}(t)\right)\right)+\frac{M_{\mathrm{HL}} K_{\mathrm{HL}}}{\left(m_{\mathrm{H}}+N m_{\mathrm{p}}\right) r_{\mathrm{H}}^{2}} X_{\mathrm{HL}}+\frac{M_{\mathrm{HL}} K_{\mathrm{HL}}}{m_{\mathrm{L}} r_{\mathrm{L}}^{2}} X_{\mathrm{HL}}+\frac{M_{\mathrm{HL}} r_{\mathrm{H}} k_{\mathrm{r} 2 \mathrm{~L}}}{r_{\mathrm{L}} m_{\mathrm{L}} r_{\mathrm{r} 2 \mathrm{~b}}^{2}} X_{\mathrm{r} 2 \mathrm{~L}}=\frac{M_{\mathrm{HL}} r_{\mathrm{H}}}{r_{\mathrm{L}} m_{\mathrm{L}}} P_{\mathrm{L}} .
\end{aligned}
$$

3.5. Dimensionless Dynamical Equation. It is important to perform dimensionless processing of nonlinear differential equations before solving them by the numerical method. $\omega_{\mathrm{n}}$ was defined as follows:

$$
\omega_{\mathrm{n}}=\sqrt{\frac{K_{\mathrm{sp}}}{M_{\mathrm{sp}}}},
$$

where $K_{\mathrm{sp}}$ is the average meshing stiffness of differential stage external meshing.

The dimensionless time-independent variable $(\tau)$ and the dimensionless exciting frequency $(\Omega)$ were defined as follows:

$$
\left\{\begin{array}{l}
\tau=\omega_{\mathrm{n}} t, \\
\Omega=\frac{\omega}{\omega_{\mathrm{n}}},
\end{array}\right.
$$

where $\omega$ is the exciting frequency of the system.
Now, by defining $b_{c}$ as the displacement nominal scale, the dimensionless displacement vector $\bar{X}$ was expressed by the following equation:

$$
\bar{X}(\tau)=\frac{X\left(\tau / \omega_{\mathrm{n}}\right)}{b_{\mathrm{c}}} .
$$

$C_{i j}, K_{i j}$, and $P_{i}$ denote the damping coefficient, meshing stiffness, and load, respectively in equations (12)-(18); hence, their corresponding $\bar{C}_{i j}, \bar{K}_{i j}$, and $\bar{P}_{i}$ after dimensionless calculation were expressed as follows:

$$
\left\{\begin{array}{l}
\bar{C}_{i j}=\frac{C_{i j}}{\omega_{\mathrm{n}}}, \\
\bar{K}_{i j}=\frac{K_{i j}}{\omega_{\mathrm{n}}^{2}}, \\
\bar{P}_{i}=\frac{P_{i}}{\left(b_{\mathrm{c}} \omega_{\mathrm{n}}^{2}\right)} .
\end{array}\right.
$$


Therefore,

$$
\begin{aligned}
& M_{\mathrm{sp}} \ddot{\bar{X}}_{\mathrm{sp} i}+\frac{M_{\mathrm{sp}}}{m_{\mathrm{s} 1}} \sum_{i=1}^{N}\left(\bar{K}_{\mathrm{sp} i}(\tau) f\left(\bar{X}_{\mathrm{sp} i}, \bar{b}_{\mathrm{sp} i}\right)+\bar{C}_{\mathrm{sp}} \dot{\bar{X}}_{\mathrm{sp} i}\right)+\frac{M_{\mathrm{sp}}}{m_{\mathrm{p}}}\left(K_{\mathrm{sp} i}(\tau) f\left(\bar{X}_{\mathrm{sp} i}, \bar{b}_{\mathrm{sp} i}\right)+\bar{C}_{\mathrm{sp}} \dot{\bar{X}}_{\mathrm{sp} i}\right) \\
& -\frac{M_{\mathrm{sp}}}{m_{\mathrm{p}}}\left(\bar{K}_{\mathrm{rp} i}(\tau) f\left(\bar{X}_{\mathrm{s} 1 \mathrm{r} 1}-\bar{X}_{\mathrm{sp} i}-\bar{e}_{\mathrm{sp} i}(\tau)-\bar{e}_{\mathrm{rp} i}(\tau), \bar{b}_{\mathrm{rp} i}\right)+\bar{C}_{\mathrm{rp}}\left(\dot{\bar{X}}_{\mathrm{s} 1 \mathrm{r} 1}-\dot{\bar{X}}_{\mathrm{sp} i}-\dot{\bar{e}}_{\mathrm{sp} i}(\tau)-\dot{\bar{e}}_{\mathrm{rp} i}(\tau)\right)\right)=\frac{M_{\mathrm{sp}}}{m_{\mathrm{s} 1} b_{c} \omega_{\mathrm{n}}^{2}} P_{\mathrm{D}}-M_{\mathrm{sp}} \ddot{\bar{e}}_{\mathrm{sp} i}(\tau),
\end{aligned}
$$

$$
\begin{aligned}
& M_{\mathrm{s} 1 \mathrm{r} 1} \ddot{\bar{X}}_{\mathrm{s} 1 \mathrm{r} 1}+\frac{M_{\mathrm{s} 1 \mathrm{r} 1}}{m_{\mathrm{s} 1}} \sum_{i=1}^{N}\left(\bar{K}_{\mathrm{sp} i}(\tau) f\left(\bar{X}_{\mathrm{sp} i}, \bar{b}_{\mathrm{sp} i}\right)+\bar{C}_{\mathrm{sp}} \dot{\bar{X}}_{\mathrm{sp} i}\right)+\frac{M_{\mathrm{s} 1 \mathrm{r} 1}}{m_{\mathrm{r} 1}} \sum_{i=1}^{N}\left(\bar{K}_{\mathrm{rp} i}(\tau) f\left(\bar{X}_{\mathrm{s} 1 \mathrm{r} 1}-\bar{X}_{\mathrm{sp} i}-\bar{e}_{\mathrm{sp} i}(\tau)-\bar{e}_{\mathrm{rp} i}(\tau), \bar{b}_{\mathrm{rp} i}\right)\right. \\
& \left.+\bar{C}_{\mathrm{rp}}\left(\dot{\bar{X}}_{\mathrm{s} 1 \mathrm{r} 1}-\dot{\bar{X}}_{\mathrm{sp} i}-\dot{\bar{e}}_{\mathrm{spi}}(\tau)-\dot{\bar{e}}_{\mathrm{rpi}}(\tau)\right)\right)-\frac{M_{\mathrm{s} 1 \mathrm{r} 1} K_{\mathrm{r} 1 \mathrm{~s} 2}}{m_{\mathrm{r} 1} r_{\mathrm{r} 1 \mathrm{~b}}^{2} \omega_{\mathrm{n}}^{2}} \bar{X}_{\mathrm{r} 1 \mathrm{~s} 2}=\frac{M_{\mathrm{s} 1 \mathrm{r} 1}}{m_{\mathrm{s} 1} b_{c} \omega_{\mathrm{n}}^{2}} P_{\mathrm{D}},
\end{aligned}
$$

$$
\begin{gathered}
M_{\mathrm{r} 1 \mathrm{~s} 2} \ddot{\bar{X}}_{\mathrm{r} 1 \mathrm{~s} 2}-\frac{M_{\mathrm{r} 1 \mathrm{~s} 2}}{m_{\mathrm{r} 1}} \sum_{i=1}^{N}\left(\bar{K}_{\mathrm{rp} i}(\tau) f\left(\bar{X}_{\mathrm{s} 1 \mathrm{r} 1}-\bar{X}_{\mathrm{sp} i}-\bar{e}_{\mathrm{sp} i}(\tau)-\bar{e}_{\mathrm{rp} i}(\tau), \bar{b}_{\mathrm{rp} i}\right)+\bar{C}_{\mathrm{rp}}\left(\dot{\bar{X}}_{\mathrm{s} 1 \mathrm{r} 1}-\dot{\bar{X}}_{\mathrm{spi}}-\dot{\bar{e}}_{\mathrm{spi} i}(\tau)-\dot{\bar{e}}_{\mathrm{rpi} i}(\tau)\right)\right) \\
+\frac{M_{\mathrm{r} 1 \mathrm{2} 2} K_{\mathrm{r} 1 \mathrm{~s} 2}}{m_{\mathrm{r} 1} r_{\mathrm{r} 1 \mathrm{~b}}^{2} \omega_{\mathrm{n}}^{2}} \bar{X}_{\mathrm{r} 1 \mathrm{~s} 2}-\frac{r_{\mathrm{r} 1 \mathrm{~b}}}{r_{\mathrm{s} 2 \mathrm{~b}}} \frac{M_{\mathrm{r} 1 \mathrm{~s} 2}}{m_{\mathrm{s} 2}} \sum_{j=1}^{M}\left(\bar{K}_{\mathrm{sm} j}(\tau) f\left(\bar{X}_{\mathrm{sm} j}, \bar{b}_{\mathrm{sm} j}\right)+\bar{C}_{\mathrm{sm}} \dot{\bar{X}}_{\mathrm{sm} j}\right)+\frac{M_{\mathrm{r} 122} K_{\mathrm{r} 1 \mathrm{~s} 2}}{m_{\mathrm{s} 2} r_{\mathrm{s} 2 \mathrm{~b}}^{2} \omega_{\mathrm{n}}^{2}} X_{\mathrm{r} 1 \mathrm{~s} 2}=0,
\end{gathered}
$$

$$
\begin{gathered}
M_{\mathrm{sm}} \ddot{\bar{X}}_{\mathrm{sm} j}+\frac{M_{\mathrm{sm}}}{m_{\mathrm{s} 2}} \sum_{j=1}^{M}\left(\bar{K}_{\mathrm{sm} j}(\tau) f\left(\bar{X}_{\mathrm{sm} j}, \bar{b}_{\mathrm{sm} j}\right)+\bar{C}_{\mathrm{sm}} \dot{\bar{X}}_{\mathrm{sm} j}\right)-\frac{M_{\mathrm{sm}} K_{\mathrm{r} 1 \mathrm{~s} 2}}{m_{\mathrm{s} 2} r_{\mathrm{r} 1 \mathrm{~b}} r_{\mathrm{s} 2 \mathrm{~b}} \omega_{\mathrm{n}}^{2}} \bar{X}_{\mathrm{r} 1 \mathrm{~s} 2}+\frac{M_{\mathrm{sm}}}{m_{\mathrm{m}}}\left(\bar{K}_{\mathrm{sm} j}(\tau) f\left(\bar{X}_{\mathrm{sm} j}, \bar{b}_{\mathrm{sm} j}\right)+\bar{C}_{\mathrm{sm}} \dot{\bar{X}}_{\mathrm{sm} j}\right) \\
-\frac{M_{\mathrm{sm}}}{m_{\mathrm{m}}}\left(\bar{K}_{\mathrm{rm} j}(\tau) f\left(\bar{X}_{\mathrm{s} 2 \mathrm{r} 2}-\bar{X}_{\mathrm{sm} j}-\bar{e}_{\mathrm{sm} j}(\tau)-\bar{e}_{\mathrm{rm} j}(\tau), \bar{b}_{\mathrm{rm} j}\right)+\bar{C}_{\mathrm{rm}}\left(\dot{\bar{X}}_{\mathrm{s} 2 \mathrm{r} 2}-\dot{\bar{X}}_{\mathrm{sm} j}-\dot{\bar{e}}_{\mathrm{sm} j}(\tau)-\dot{\bar{e}}_{\mathrm{rm} j}(\tau)\right)\right)=-M_{\mathrm{sm}} \ddot{\bar{e}}_{\mathrm{sm} j}(\tau),
\end{gathered}
$$

$$
\begin{aligned}
& M_{\mathrm{s} 2 \mathrm{r} 2} \ddot{\bar{X}}_{\mathrm{s} 2 \mathrm{r} 2}+\frac{M_{\mathrm{s} 2 \mathrm{r} 2}}{m_{\mathrm{s} 2}} \sum_{j=1}^{M}\left(\bar{K}_{\mathrm{sm} j}(\tau) f\left(\bar{X}_{\mathrm{sm} j}, \bar{b}_{\mathrm{sm} j}\right)+\bar{C}_{\mathrm{sm}} \dot{\bar{X}}_{\mathrm{sm} j}\right)-\frac{M_{\mathrm{s} 2 \mathrm{r} 2} K_{\mathrm{r} 1 \mathrm{~s} 2}}{m_{\mathrm{s} 2} r_{\mathrm{r} 1 \mathrm{~b}} r_{\mathrm{s} 2 \mathrm{~b}} \omega_{\mathrm{n}}^{2}} \bar{X}_{\mathrm{r} 1 \mathrm{~s} 2} \\
& \quad+\frac{M_{\mathrm{s} 2 \mathrm{r} 2}}{m_{\mathrm{r} 2}} \sum_{j=1}^{M}\left(\bar{K}_{\mathrm{rm} j}(\tau) f\left(\bar{X}_{\mathrm{s} 2 \mathrm{r} 2}-\bar{X}_{\mathrm{sm} j}-\bar{e}_{\mathrm{sm} j}(\tau)-\bar{e}_{\mathrm{rm} j}(\tau), \bar{b}_{\mathrm{rm} j}\right)+\bar{C}_{\mathrm{rm}}\left(\dot{\bar{X}}_{\mathrm{s} 2 \mathrm{r} 2}-\dot{\bar{X}}_{\mathrm{sm} j}-\dot{\bar{e}}_{\mathrm{sm} j}(\tau)-\dot{\bar{e}}_{\mathrm{rm} j}(\tau)\right)\right) \\
& \quad-\frac{M_{\mathrm{s} 2 \mathrm{r} 2} K_{\mathrm{r} 2 \mathrm{~L}}}{m_{\mathrm{r} 2} r_{\mathrm{r} 2 \mathrm{~b}}^{2} \omega_{\mathrm{n}}^{2}} \overline{\mathrm{r}}_{\mathrm{r} 2 \mathrm{~L}}=0,
\end{aligned}
$$

$$
\begin{aligned}
& M_{\mathrm{r} 2 \mathrm{~L}} \ddot{\bar{X}}_{\mathrm{r} 2 \mathrm{~L}}-\frac{M_{\mathrm{r} 2 \mathrm{~L}}}{m_{\mathrm{r} 2}} \sum_{j=1}^{M}\left(\bar{K}_{\mathrm{rm} j}(\tau) f\left(\bar{X}_{\mathrm{s} 2 \mathrm{r} 2}-\bar{X}_{\mathrm{sm} j}-\bar{e}_{\mathrm{sm} j}(\tau)-\bar{e}_{\mathrm{rm} j}(\tau), \bar{b}_{\mathrm{rm} j}\right)+\bar{C}_{\mathrm{rm}}\left(\dot{\bar{X}}_{\mathrm{s} 2 \mathrm{r} 2}-\dot{\bar{X}}_{\mathrm{sm} j}-\dot{\bar{e}}_{\mathrm{sm} j}(\tau)-\dot{\bar{e}}_{\mathrm{rm} j}(\tau)\right)\right) \\
& \quad+\frac{M_{\mathrm{r} 2 \mathrm{~L}} K_{\mathrm{r} 2 \mathrm{~L}}}{m_{\mathrm{r} 2} 2_{\mathrm{r} 2 \mathrm{~b}}^{2} \omega_{\mathrm{n}}^{2}} \bar{X}_{\mathrm{r} 2 \mathrm{~L}}+\frac{M_{\mathrm{r} 2 \mathrm{~L}} r_{\mathrm{r} 2 \mathrm{~b}} K_{\mathrm{HL}}}{m_{\mathrm{L}} r_{\mathrm{H}} r_{\mathrm{L}}^{2} \omega_{\mathrm{n}}^{2}} \bar{X}_{\mathrm{HL}}+\frac{M_{\mathrm{r} 2 \mathrm{~L}} k_{\mathrm{r} 2 \mathrm{~L}}}{r_{\mathrm{L}} m_{\mathrm{L}} r_{\mathrm{r} 2 \mathrm{~b}} \omega_{\mathrm{n}}^{2}} \bar{X}_{\mathrm{r} 2 \mathrm{~L}}=\frac{M_{\mathrm{r} 2 \mathrm{~L}} r_{\mathrm{r} 2 \mathrm{~b}}}{r_{\mathrm{L}} m_{\mathrm{L}} b_{c} \omega_{\mathrm{n}}^{2}} P_{\mathrm{L}},
\end{aligned}
$$

$$
\begin{aligned}
& M_{\mathrm{HL}} \ddot{\bar{X}}_{\mathrm{HL}}-\frac{M_{\mathrm{HL}}}{\left(m_{\mathrm{H}}+N m_{\mathrm{p}}\right)} \sum_{i=1}^{N}\left(\bar{K}_{\mathrm{spi}}(\tau) f\left(\bar{X}_{\mathrm{sp} i}, \bar{b}_{\mathrm{sp} i}\right)+\bar{C}_{\mathrm{sp}} \dot{\bar{X}}_{\mathrm{sp} i}+\bar{K}_{\mathrm{rp} i}(\tau) f\left(\bar{X}_{\mathrm{s} 1 \mathrm{r} 1}-\bar{X}_{\mathrm{spi} i}-\bar{e}_{\mathrm{sp} i}(\tau)-\bar{e}_{\mathrm{rp} i}(\tau), \bar{b}_{\mathrm{rp} i}\right)\right. \\
& \left.\quad+\bar{C}_{\mathrm{rp}}\left(\dot{\bar{X}}_{\mathrm{s} 1 \mathrm{r} 1}-\dot{\bar{X}}_{\mathrm{spi}}-\dot{\bar{e}}_{\mathrm{spi}}(\tau)-\dot{\bar{e}}_{\mathrm{rpi}}(\tau)\right)\right)+\frac{M_{\mathrm{HL}} K_{\mathrm{HL}}}{\left(m_{\mathrm{H}}+N m_{\mathrm{p}}\right) r_{\mathrm{H}}^{2} \omega_{\mathrm{n}}^{2}} \bar{X}_{\mathrm{HL}}+\frac{M_{\mathrm{HL}} K_{\mathrm{HL}}}{m_{\mathrm{L}} r_{\mathrm{L}}^{2} \omega_{\mathrm{n}}^{2}} \bar{X}_{\mathrm{HL}}+\frac{M_{\mathrm{HL}} r_{\mathrm{H}} k_{\mathrm{r} 2 \mathrm{~L}}}{r_{\mathrm{L}} m_{\mathrm{L}} \overline{\mathrm{X}}_{\mathrm{r} 2 \mathrm{~b}}^{2} \omega_{\mathrm{n}}^{2}}=\frac{M_{\mathrm{HL}} r_{\mathrm{H}}}{r_{\mathrm{L}} m_{\mathrm{L}} b_{\mathrm{c}} \omega_{\mathrm{n}}^{2}} P_{\mathrm{L}} .
\end{aligned}
$$




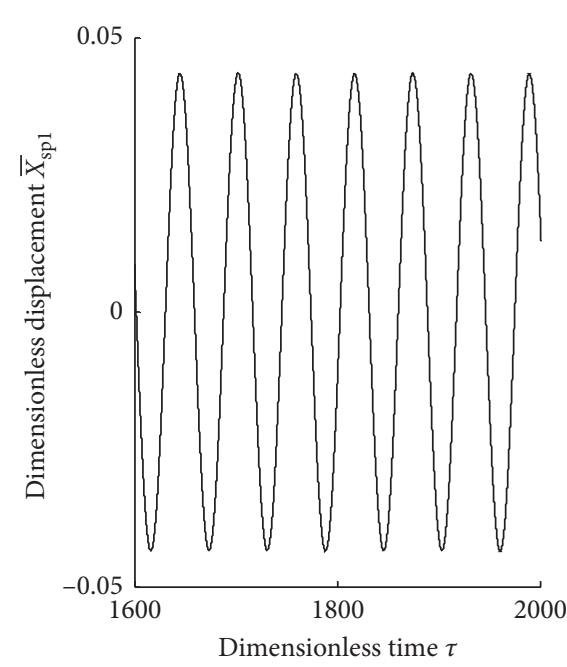

(a)

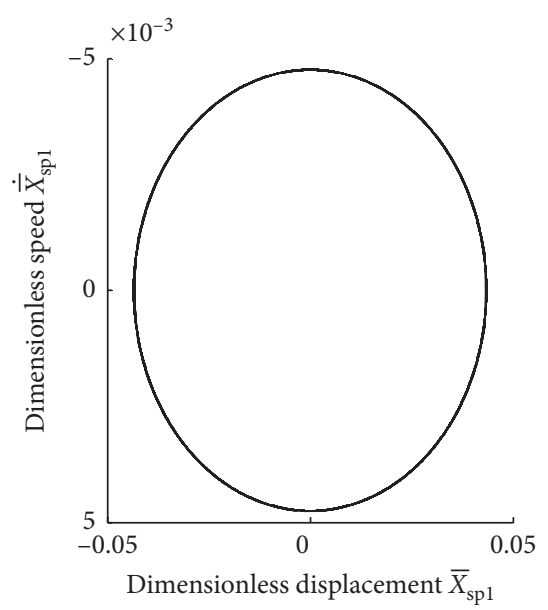

(c)

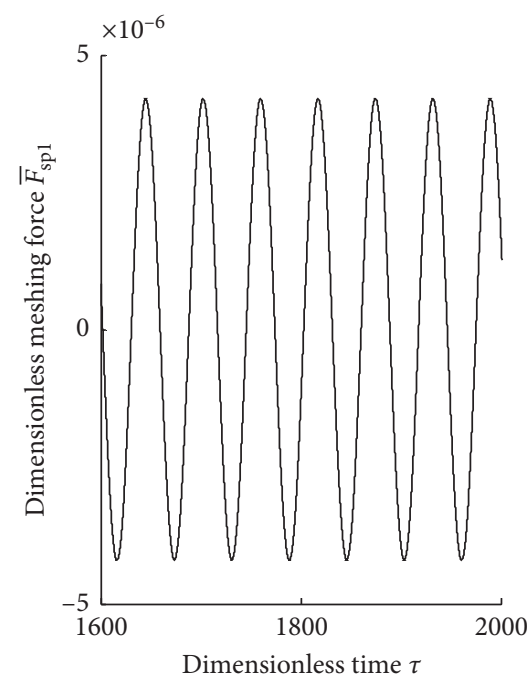

(b)

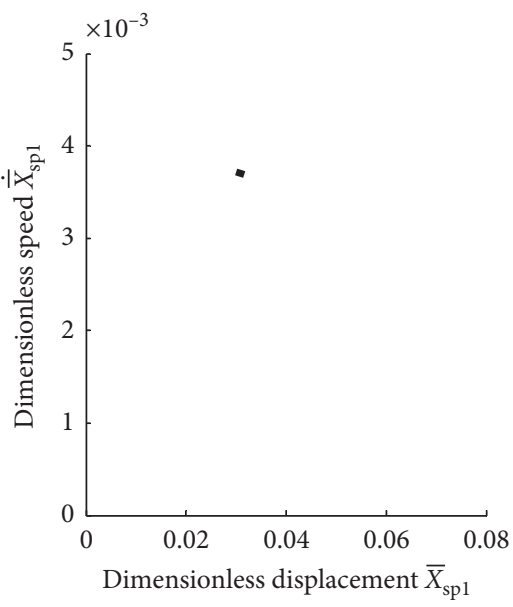

(d)

Figure 3: Process diagrams of response, phase diagram, and Poincaré section under $b=0 \mu \mathrm{m}$ for differential stage. (a) Time process diagram of displacement response. (b) Time process diagram of meshing force response. (c) Phase diagram. (d) Poincaré section.

\section{Analysis of Dynamic Characteristics}

The dimensionless nonlinear dynamic equations of encased differential planetary gear train are expressed in equations (23)-(29), and the Newmark direct integral method of unconditional convergence was employed to solve them. The impacts of backlash on dynamic characteristics of gear train were analyzed by time process diagrams of displacement response and meshing force response, phase diagram, and Poincaré section.

In our research, an encased differential planetary train reducer was analyzed based on following basic parameters. For differential stage: gear modulus $\left(m_{1}\right)=7 \mathrm{~mm}$, number of planetary gear $(N)=3$, number of tooth on sun gear $\left(Z_{s 1}\right)=$ 37 , number of tooth on planetary gear $\left(Z_{\mathrm{p}}\right)=56$, number of tooth on internal gear $\left(Z_{\mathrm{rl}}\right)=149$, coefficient of tooth width $\left(\phi_{\mathrm{d} 1}\right)=1.2$, pressure angle $\left(\alpha_{\mathrm{n} 1}\right)=20^{\circ}$, and for encased stage, gear modulus $\left(m_{2}\right)=7 \mathrm{~mm}$, number of star gear $(M)=5$, number of tooth on sun gear $\left(Z_{\mathrm{s} 2}\right)=71$, number of tooth on star gear $\left(Z_{\mathrm{m}}\right)=39$, number of tooth on internal gear $\left(Z_{\mathrm{r} 2}\right)=$ 149 , coefficient of tooth width $\left(\phi_{\mathrm{d} 2}\right)=0.6$, and pressure angle $\left(\alpha_{\mathrm{n} 2}\right)=20^{\circ}$. Moreover, input shaft speed $(n)=3000 \mathrm{r} / \mathrm{min}$, input power $P=20000 \mathrm{~kW}$, support stiffness of sun gear for differential and encased stages $=2 \times 10^{8} \mathrm{~N} \cdot \mathrm{m}^{-1}$, support stiffness of planetary gear and star gear $=10^{9} \mathrm{~N} \cdot \mathrm{m}^{-1}$, and comprehensive error $=30 \mu \mathrm{m}$.

In order to simplify the analysis, it was assumed that the size of backlash of internal and external meshing was the same for each stage, and the backlash of differential and encased stages was denoted as $b_{1}$ and $b_{2}$, respectively.

\subsection{Impacts of Simultaneous Change of Two-Stage Backlash on} Dynamic Characteristics of Gear Train. It was assumed that the size of two-stage backlash of internal and external meshing was the same, and the two-stage process diagram of response, phase diagram, and Poincaré section was obtained for backlashes of $0 \mu \mathrm{m}, 20 \mu \mathrm{m}$, and $70 \mu \mathrm{m}$ (Figures 3-8). 


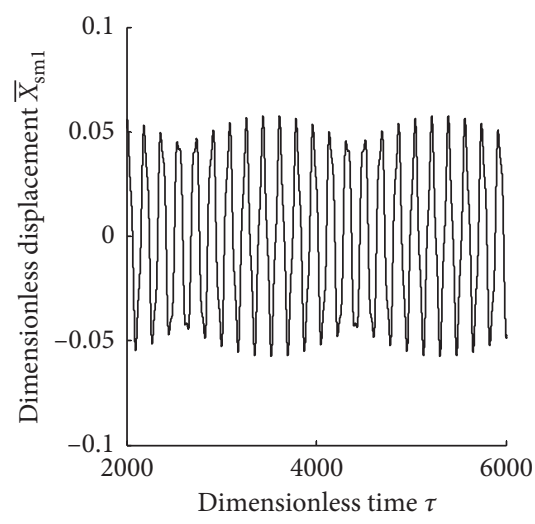

(a)

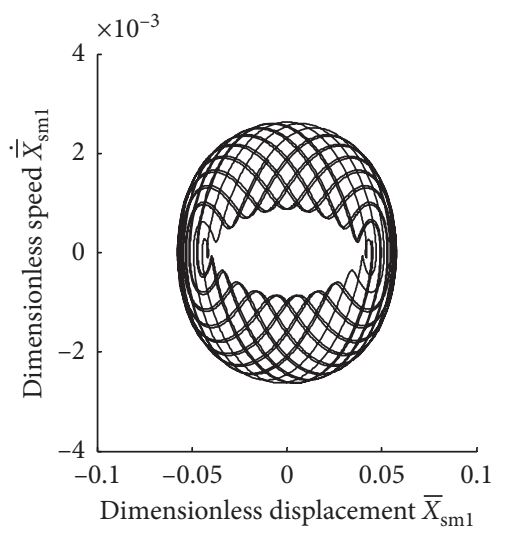

(c)

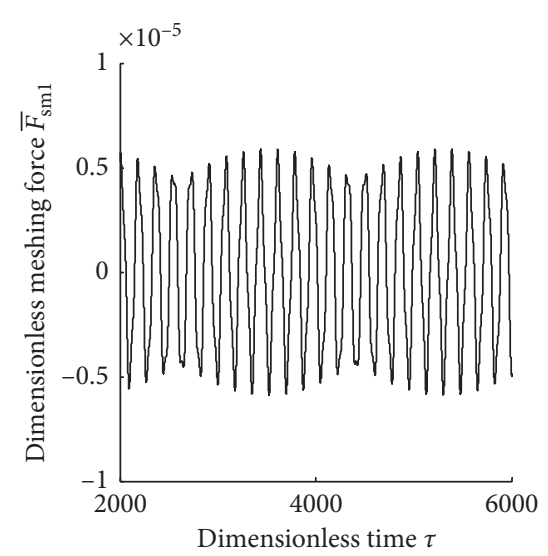

(b)

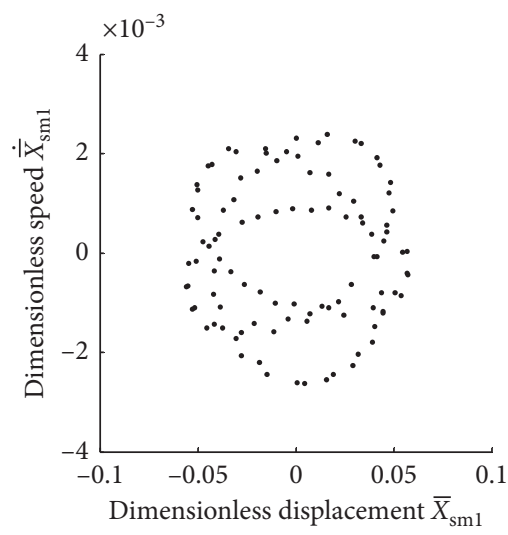

(d)

Figure 4: Process diagrams of response, phase diagram, and Poincaré section under $b=0 \mu \mathrm{m}$ for encased stage. (a) Time process diagram of displacement response. (b) Time process diagram of meshing force response. (c) Phase diagram. (d) Poincaré section.

4.1.1. Two-Stage dynamic response for $0 \mu m$ Backlash. When the value of backlash was $0 \mu \mathrm{m}$, gear teeth were closely fitted; hence, tooth surface impact did not occur. In the differential stage, time process diagrams of displacement response and meshing force manifested periodicity. It was evident from Figure 3 that the phase diagram was a closed loop, and the Poincare section was a point; therefore a simple harmonic periodic gear motion was developed in the differential stage. Figure 4 reveals that the time process diagrams of displacement response and meshing force for encased stage also had periodicity. Moreover, the phase diagram was more complex and the Poincaré section was a closed curve; hence, quasiperiodic gear motion occurred in the encased stage.

4.1.2. Two-Stage Dynamic Response for $20 \mu \mathrm{m}$ Backlash. The backlash of internal and external meshing was $20 \mu \mathrm{m}$, which was not enough to meet the meshing line displacement change caused by meshing error; hence, the two-stage meshing gear tooth experienced a bilateral shock and tooth mesh force changed dramatically (Figures 5 and 6). The change in two-stage time process diagram of displacement response was found to be irregular, and the phase diagram and the Poincare section manifested chaotic characteristics, thus resulting in a chaotic gear motion state.

4.1.3. Two-Stage Dynamic Response for $70 \mu \mathrm{m}$ Backlash. The time process diagrams of meshing force for differential and encased stages are presented in Figures 7(b) and 8(b), respectively. The dimensionless meshing force of differential and encased stages was found to be 0 ; hence, it signifies that the backlash of $70 \mu \mathrm{m}$ was enough to meet the meshing line displacement change caused by meshing error. Phase diagrams of differential and encased stages were closed curve rings with certain widths (Figures $7(\mathrm{c})$ and 8(c)), and Poincaré sections were rings of distributed points (Figures $7(\mathrm{~d})$ and $8(\mathrm{~d})$ ). Moreover, no time-varying excitation force between gear tooth was noticed; a stable quasiperiodic motion state was obtained. However, backlash cannot be too large; otherwise, it will reduce the coincidence degree of gear transmission and could not meet the gear transmission continuity conditions.

4.2. Impacts of Change in Single-Stage Backlash on Dynamic Characteristics of Gear Train. The two-stage time process diagram of response, phase diagram, and Poincaré section of 


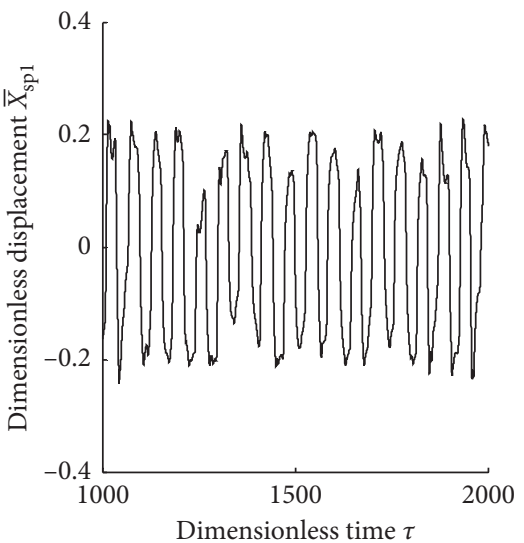

(a)

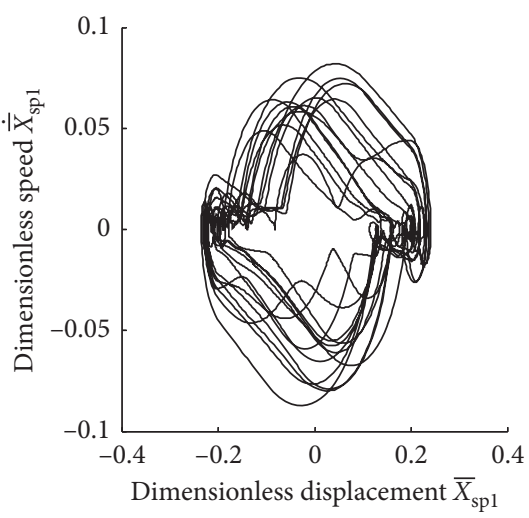

(c)

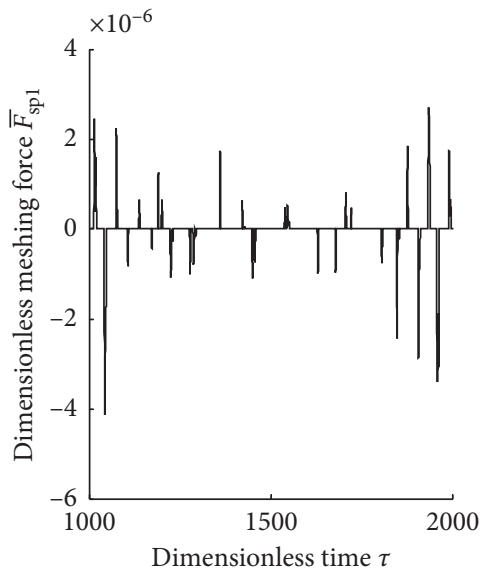

(b)

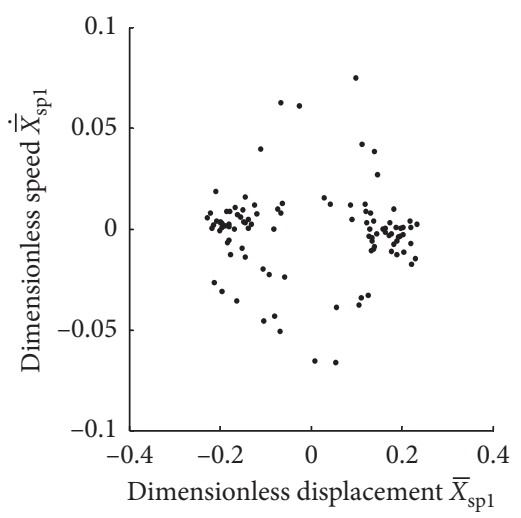

(d)

Figure 5: Process diagrams of response, phase diagram, and Poincaré section under $b=20 \mu \mathrm{m}$ for differential stage. (a) Time process diagram of displacement response. (b) Time process diagram of meshing force response. (c) Phase diagram. (d) Poincaré section.

gear train was obtained by changing the size of backlash of one stage, while keeping that of another stage unchanged (Figures 9-12). In our study, the time process diagram of response, phase diagram, and Poincaré section of gear train under the condition of $b_{1}=20 \mu \mathrm{m}$ and $b_{2}=0 \mu \mathrm{m}$ and $b_{1}=0 \mu \mathrm{m}$ and $b_{2}=20 \mu \mathrm{m}$ was compared with that of under $b_{1}=0 \mu \mathrm{m}$ and $b_{2}=0 \mu \mathrm{m}$.

(1) Two-stage dynamic response of gear train under $b_{1}=20 \mu \mathrm{m}$ and $b_{2}=0 \mu \mathrm{m}$

The two-stage time process diagram of response, phase diagram, and Poincaré section of gear train was obtained by increasing the backlash of differential stage from $0 \mu \mathrm{m}$ to $20 \mu \mathrm{m}$, while keeping that of encased stage unchanged (Figures 9 and 10). It is noticeable from Figure 9 that the meshing teeth in differential stage experienced a bilateral shock, and thus the teeth meshing force changed violently. The phase diagram and the Poincaré section of differential stage manifested chaotic characteristics, resulting in a chaotic gear motion state. In comparison to the condition $b_{1}=0 \mu \mathrm{m}$ and $b_{2}=0 \mu \mathrm{m}$, the time process diagrams of displacement response and meshing force response for encased stage under $b_{1}=20 \mu \mathrm{m}$ and $b_{2}=0 \mu \mathrm{m}$ exhibited periodicity (Figures 10(a) and 10(b)). The phase diagram and the Poincaré section for encased stage under $b_{1}=20 \mu \mathrm{m}$ and $b_{2}=0 \mu \mathrm{m}$ also yielded chaotic characteristics.

Therefore, it is obvious that the impacts of backlash change on gear force and the gear motion state in differential stage were profound, whereas the impact on gear force of encased stage was very small. Hence, it can be inferred that subtle changes in gear force can significantly change the gear motion state.

(2) Two-stage dynamic response of gear train under $b_{1}=0 \mu \mathrm{m}$ and $b_{2}=20 \mu \mathrm{m}$

The two-stage time process diagram of response, phase diagram, and Poincaré section of gear train was obtained by increasing the size of backlash in encased stage from $0 \mu \mathrm{m}$ to $20 \mu \mathrm{m}$, while keeping that of differential stage unchanged (Figures 11 and 12). It is discernible from Figure 11 that the time process diagrams of displacement response and meshing force response for differential stage were constant and also had periodicity. However, the phase diagram and the Poincaré section changed significantly. The phase diagram was a closed curve ring with a 


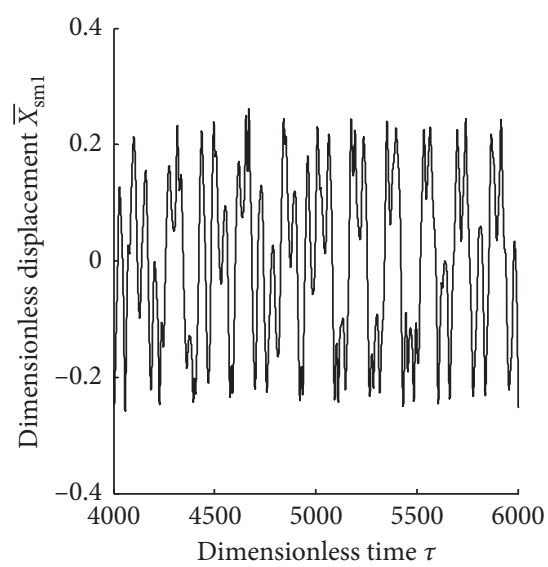

(a)

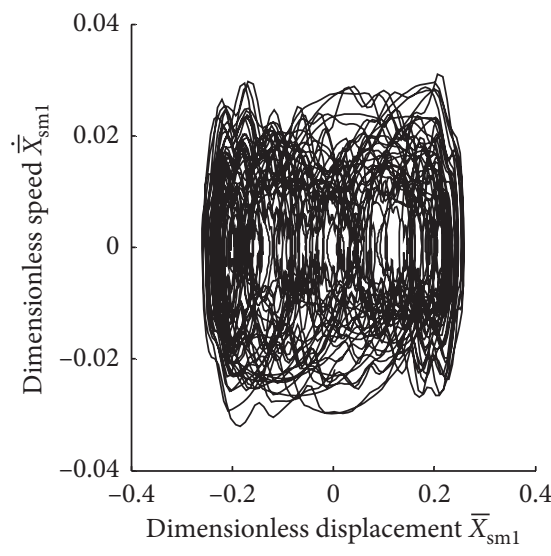

(c)

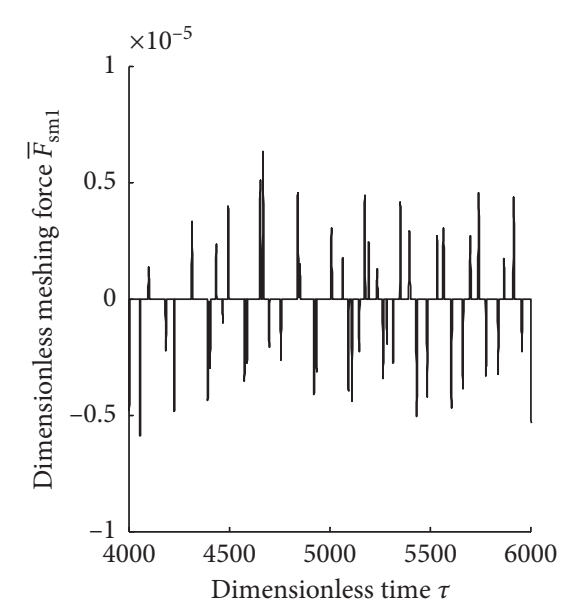

(b)

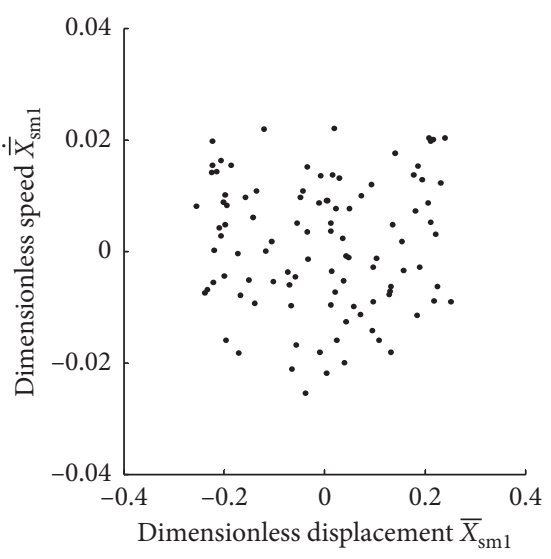

(d)

Figure 6: Process diagrams of response, phase diagram, and Poincaré section under $b=20 \mu \mathrm{m}$ for encased stage. (a) Time process diagram of displacement response. (b) Time process diagram of meshing force response. (c) Phase diagram. (d) Poincaré section.

certain width and the corresponding Poincaré section was a ring of distribution points, resulting in a stable quasiperiodic gear motion state. In comparison to Figure 4, the time process diagrams of displacement response and meshing force response of encased stage changed significantly; hence, the meshing tooth experienced a bilateral shock, and consequently, the teeth meshing force changed violently (Figure 12). The phase diagram and the Poincaré section of encased stage also manifested chaotic characteristics.

Hence, the impact of backlash change on gear force in encased stage with respect to differential stage was very small, whereas it significantly changed the gear motion state of differential stage. The impact of backlash change on gear force in encased stage with respect to its own stage was very large to change the gear motion state.

\section{Conclusion}

In this paper, a multifreedom tensional nonlinear dynamic equation of encased differential planetary gear train with multibacklash and time-varying mesh stiffness was developed. The Newmark direct integration method was employed to obtain the nonlinear dynamic response by solving the formulated nonlinear dynamic equation, and the impacts of backlash on dynamic characteristics of encased differential planetary gear train were analyzed by combining time process diagram, phase diagram, and Poincaré section. This research obtained the following conclusions:

(1) During stable motion state, no contact between intermeshing gear teeth with bigger backlash was observed. They were in a stable quasiperiodic motion state in the absence of teeth exciting force. The bilateral shock in meshing teeth was caused due to smaller backlash, thus causing dramatic changes in meshing force; hence, the gears were in a chaotic state.

(2) In order to avoid a bilateral shock in gears as well as to maintain gear teeth lubrication, a bigger backlash is required. In the absence of gear teeth exciting force, the motion became stable; thus, it reduced the intensity of vibration. However, the backlash cannot be too large; otherwise, the continuity conditions of gear transmission cannot be satisfied. 


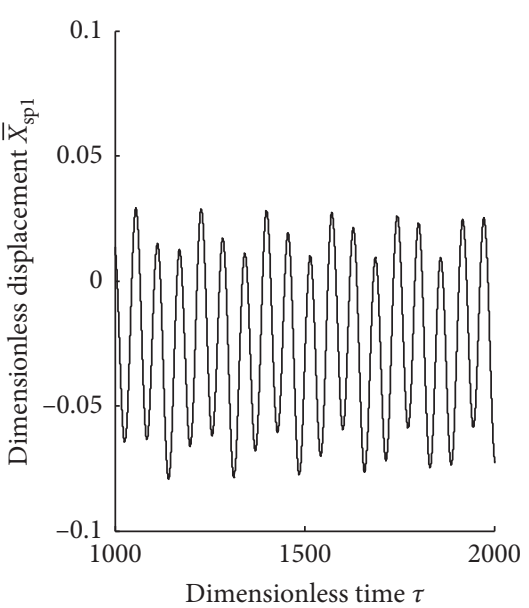

(a)

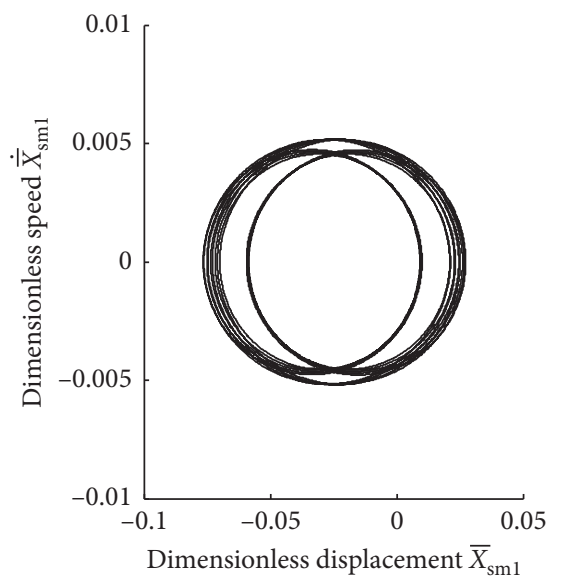

(c)

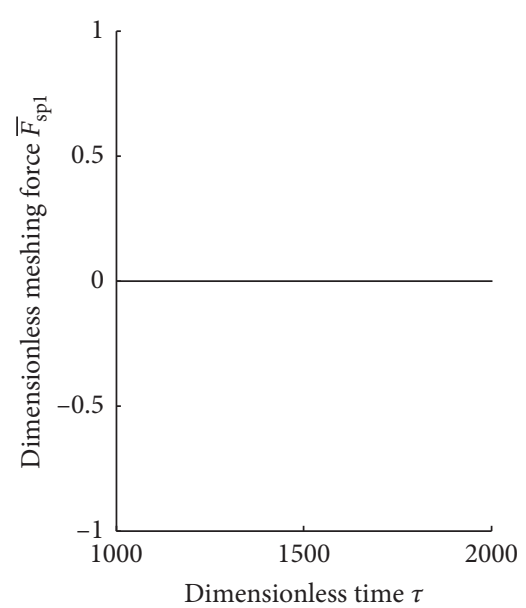

(b)

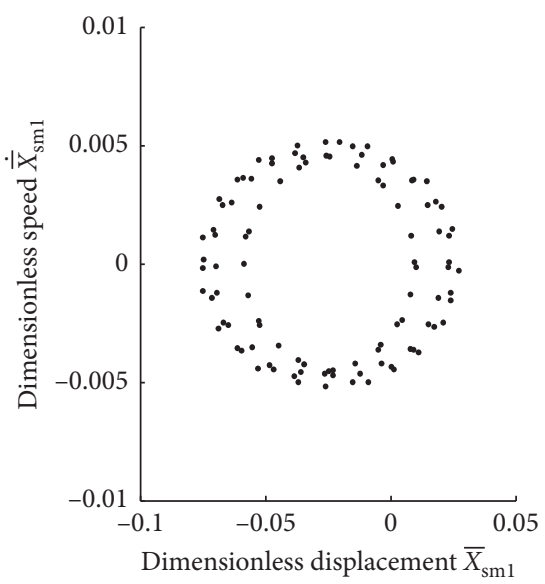

(d)

Figure 7: Process diagrams of response, phase diagram, and Poincaré section under $b=70 \mu \mathrm{m}$ for differential stage. (a) Time process diagram of displacement response. (b) Time process diagram of meshing force response. (c) Phase diagram. (d) Poincaré section.

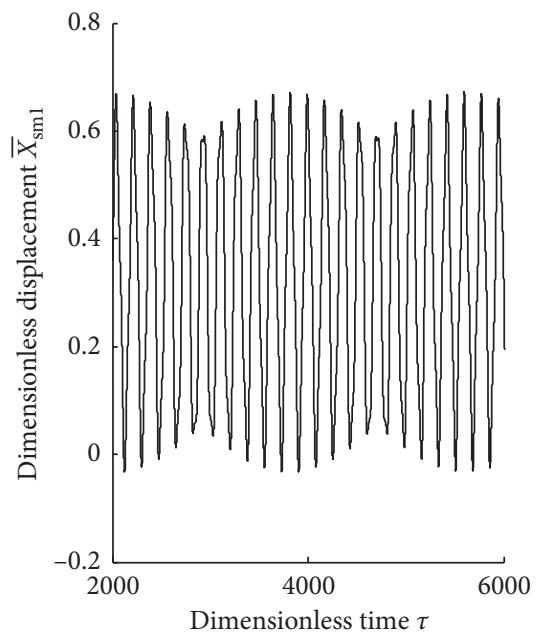

(a)

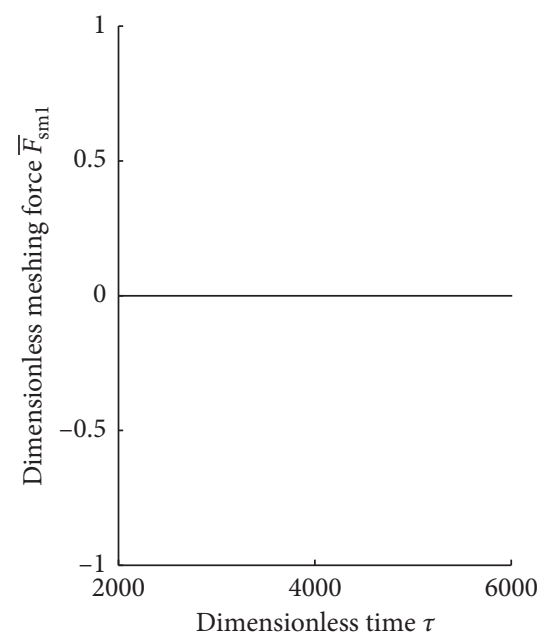

(b)

Figure 8: Continued. 


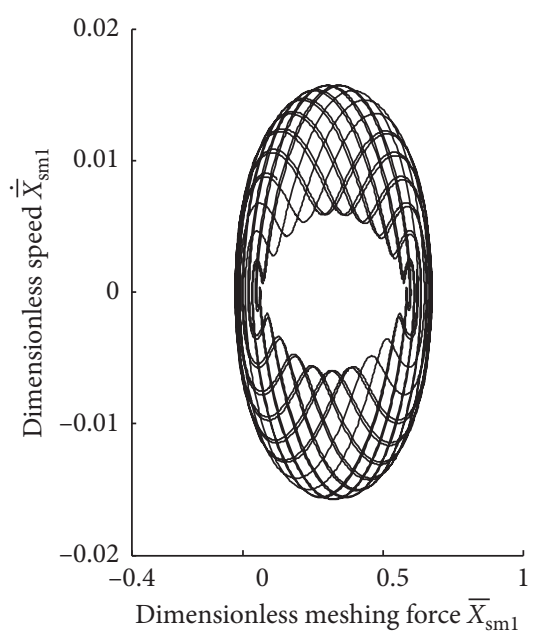

(c)

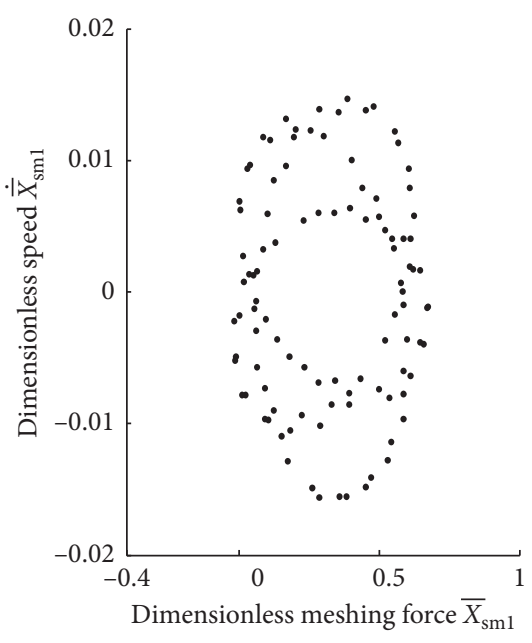

(d)

Figure 8: Process diagrams of response, phase diagram, and Poincaré section under $b=70 \mu \mathrm{m}$ for encased stage. (a) Time process diagram of displacement response. (b) Time process diagram of meshing force response. (c) Phase diagram. (d) Poincaré section.

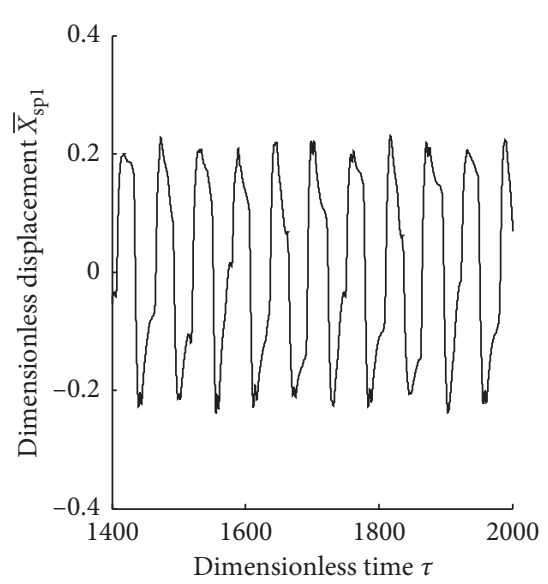

(a)

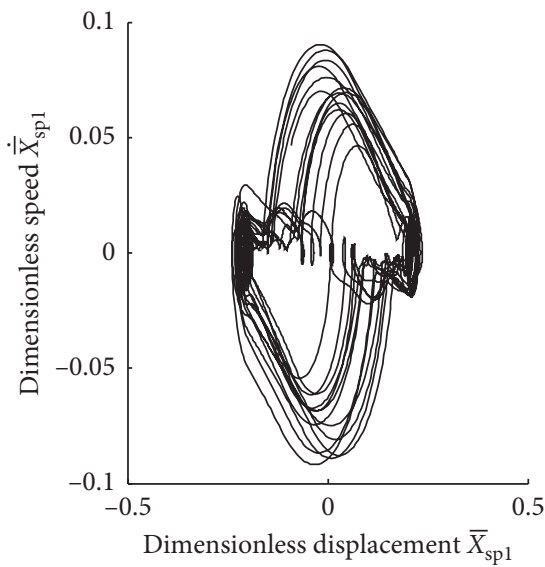

(c)

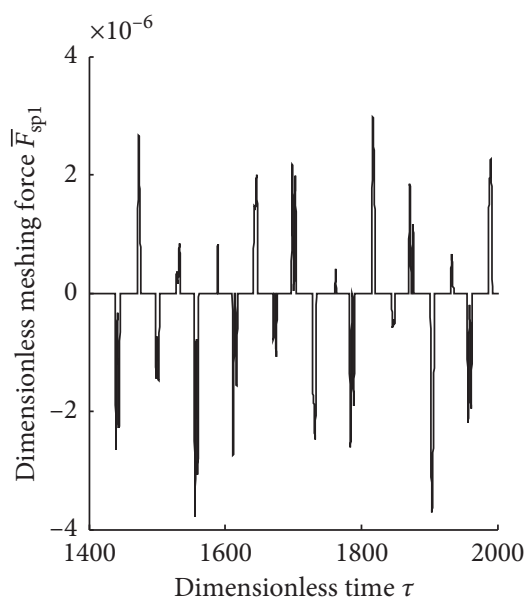

(b)

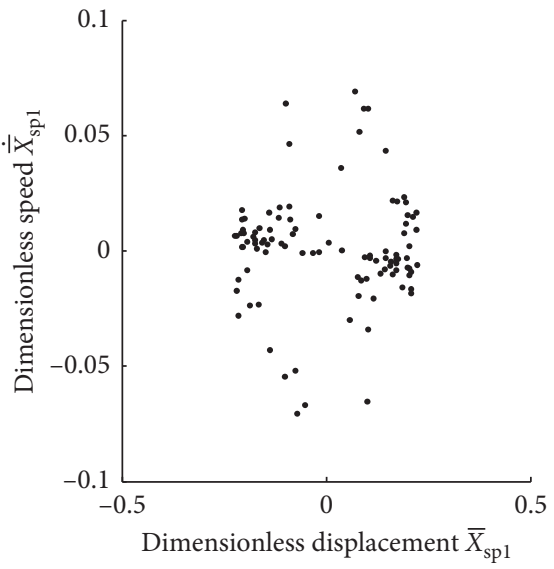

(d)

Figure 9: Process diagrams of response, phase diagram, and Poincaré section under $b_{1}=20 \mu \mathrm{m}$ and $b_{2}=0 \mu \mathrm{m}$ for differential stage. (a) Time process diagram of displacement response. (b) Time process diagram of meshing force response. (c) Phase diagram. (d) Poincaré section. 


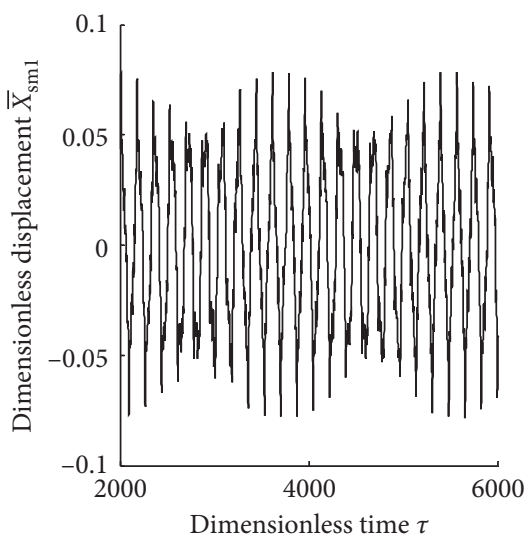

(a)

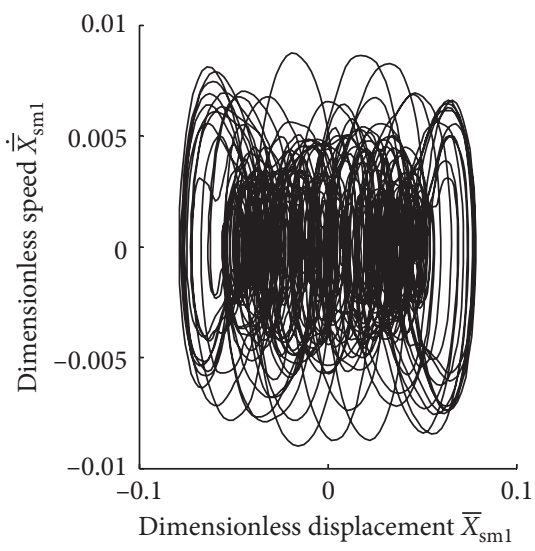

(c)

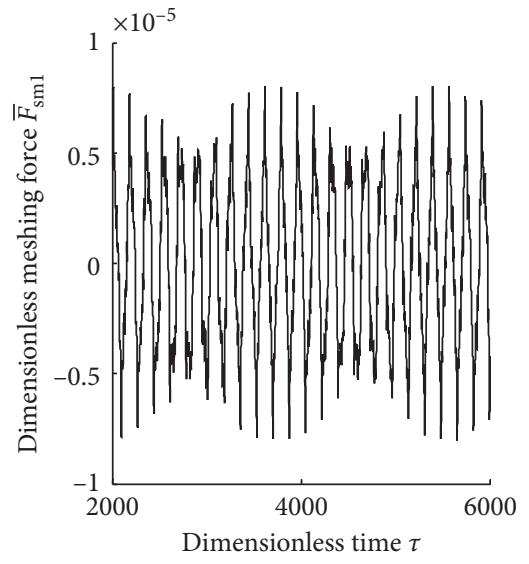

(b)

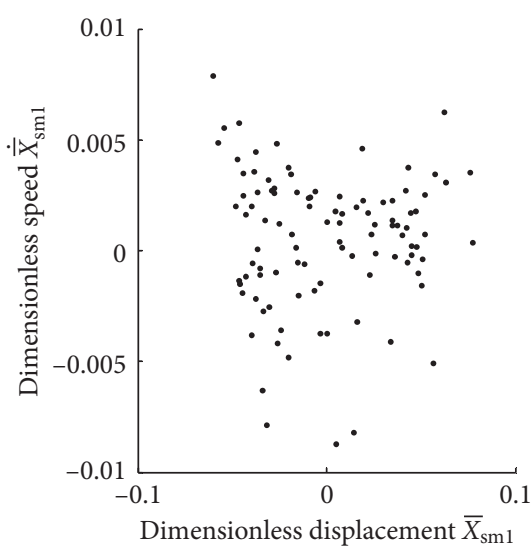

(d)

Figure 10: Process diagrams of response, phase diagram, and Poincaré section under $b_{1}=20 \mu \mathrm{m}$ and $b_{2}=0 \mu \mathrm{m}$ for encased stage. (a) Time process diagram of displacement response. (b) Time process diagram of meshing force response. (c) Phase diagram. (d) Poincaré section.

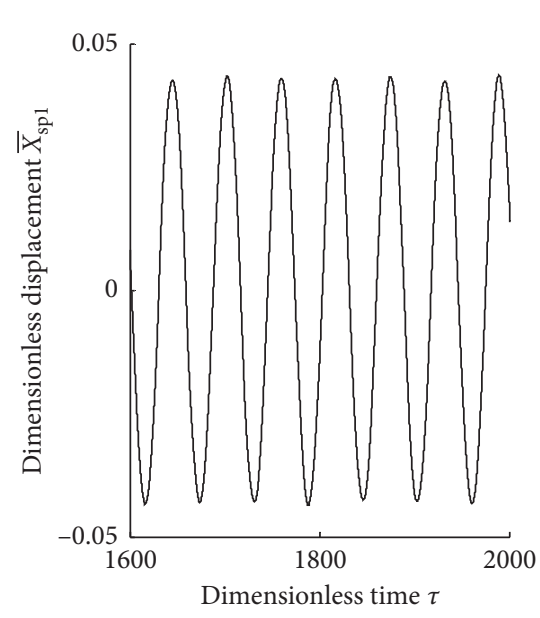

(a)

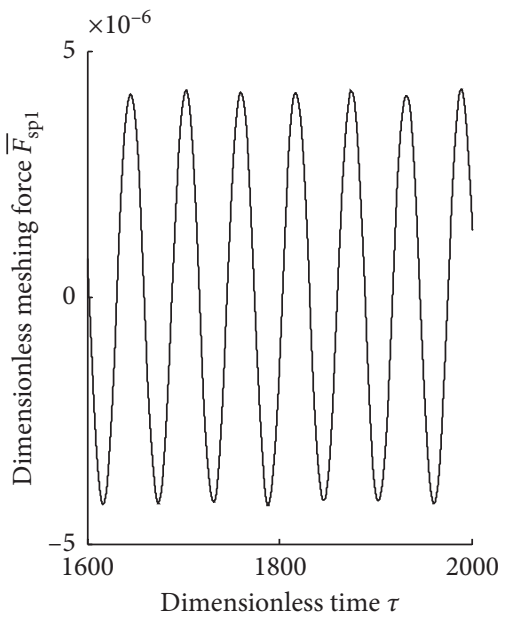

(b)

Figure 11: Continued. 


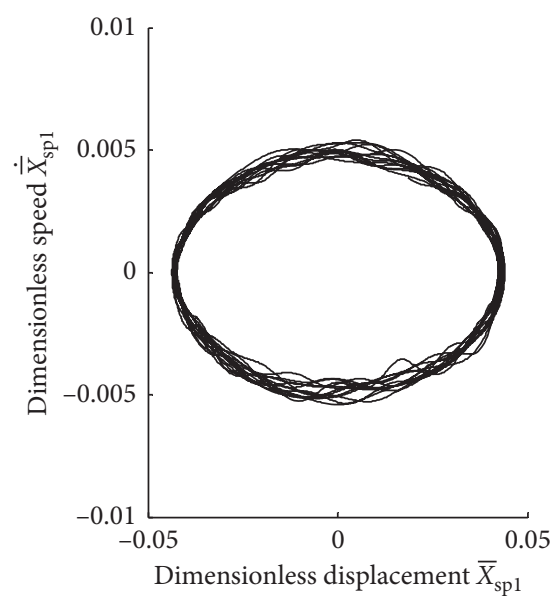

(c)

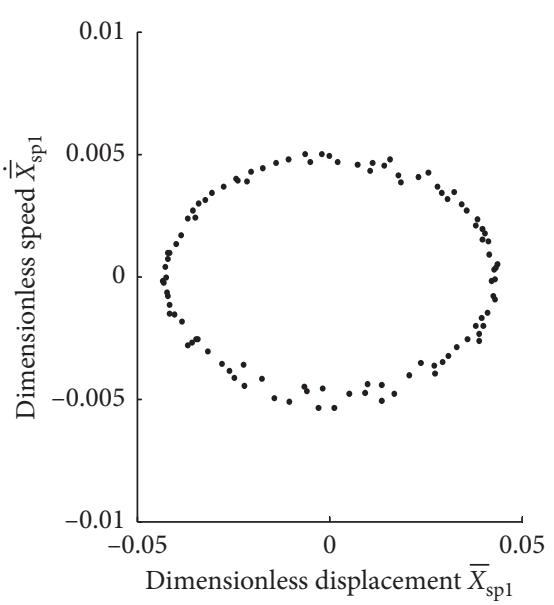

(d)

Figure 11: Process diagrams of response, phase diagram, and Poincaré section under $b_{1}=0 \mu \mathrm{m}$ and $b_{2}=20 \mu \mathrm{m}$ for differential stage. (a) Time process diagram of displacement response. (b) Time process diagram of meshing force response. (c) Phase diagram. (d) Poincaré section.

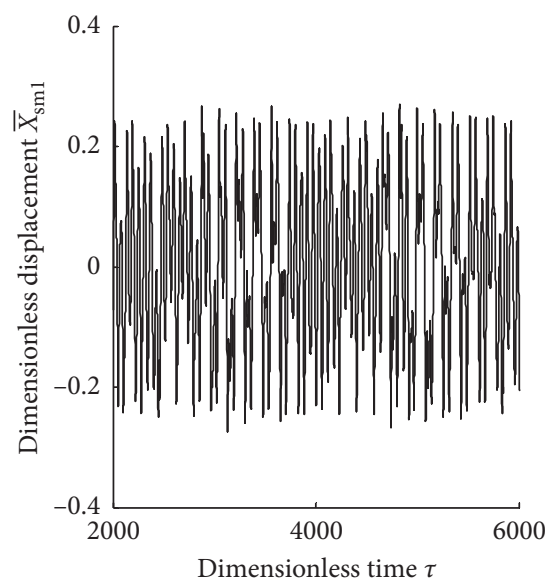

(a)

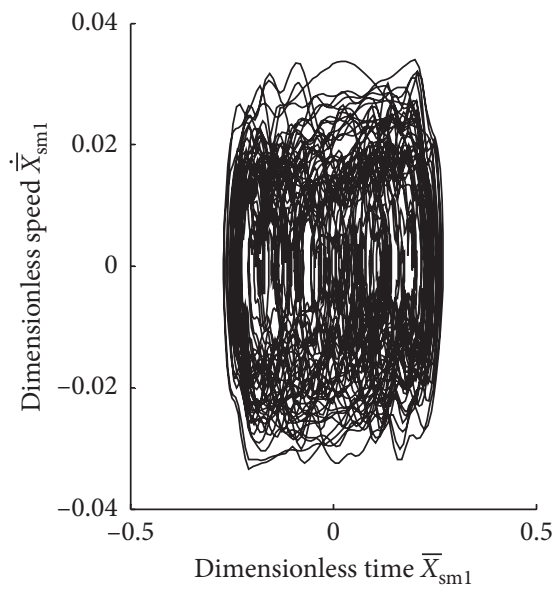

(c)

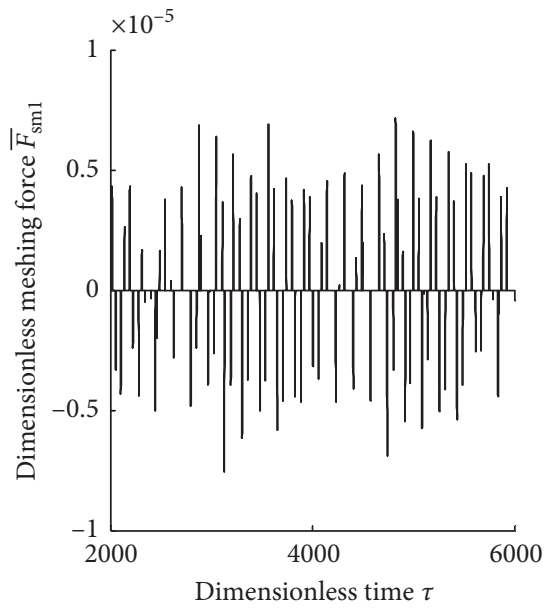

(b)

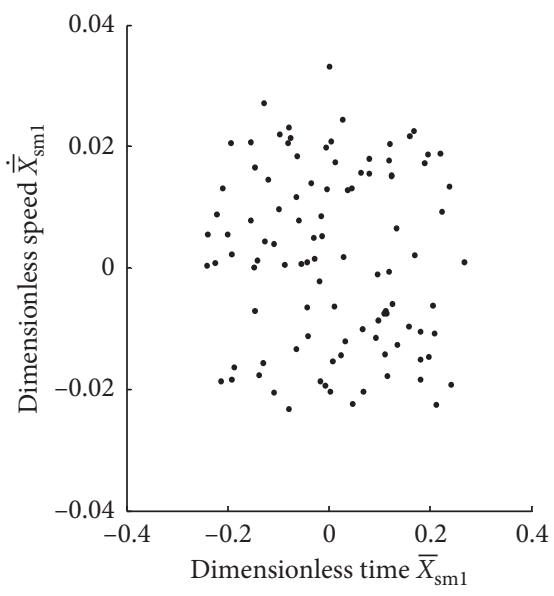

(d)

Figure 12: Process diagrams of response, phase diagram, and Poincaré section under $b_{1}=0 \mu \mathrm{m}$ and $b_{2}=20 \mu \mathrm{m}$ for encased stage. (a) Time process diagram of displacement response. (b) Time process diagram of meshing force response. (c) Phase diagram. (d) Poincaré section. 
(3) The backlash change in any transmission stage caused significant impacts on gear force and the motion state of its own stage; however, the impact on gear force of another stage was quite small. A slight change in gear force can significantly change the state of gear motion; hence, the impact of backlash change in any transmission stage on the motion state of another stage was quite large.

\section{Data Availability}

The figure data used to support the findings of this study are included within the article.

\section{Conflicts of Interest}

The authors declare that there are no conflicts of interest regarding the publication of this paper.

\section{References}

[1] R. F. Li and J. J. Wang, Dynamics of Gear System-Vibration, Impact, Noise, Science Press, Beijing, China, 1997.

[2] P. Velex and L. Flamand, "Dynamic response of planetary trains to mesh parametric excitations," Journal of Mechanical Design, vol. 118, no. 1, pp. 7-14, 1996.

[3] S. Theodossiades and S. Natsiavas, "Non-linear dynamics of gear-pair systems with periodic stiffness and backlash," Journal of Sound and Vibration, vol. 229, no. 2, pp. 287-310, 2000.

[4] J. Wang, R. Li, and X. Peng, "Survey of nonlinear vibration of gear transmission systems," Applied Mechanics Reviews, vol. 56, no. 3, pp. 309-329, 2003.

[5] A. Al-shyyab and A. Kahraman, "Non-linear dynamic analysis of a multi-mesh gear train using multi-term harmonic balance method: sub-harmonic motions:sub-harmonic motions," Journal of Sound and Vibration, vol. 279, no. 1-2, pp. 417-451, 2005.

[6] C. Q. Ma, Z. X. Cheng, W. Z. Zhang et al., Involute Planetary Gear Transmission Design, Science Press, Beijing, China, 1987.

[7] Z. M. Sun, L. H. Ji, and Y. W. Shen, "Nonlinear dynamics of $2 \mathrm{~K}-\mathrm{H}$ planetary gear train," Journal of Tsinghua University, vol. 43, pp. 636-639, 2003, (in Chinese).

[8] S. Y. Chen, J. Y. Tang, L. C. Wang, and Q. Wang, "Nonlinear dynamic characteristics of geared rotor bearing systems with dynamic backlash and friction," Mechanism and Machine Theory, vol. 46, no. 4, pp. 466-478, 2011.

[9] Z. Liu, Z. Liu, J. Zhao, and G. Zhang, "Study on interactions between tooth backlash and journal bearing clearance nonlinearity in spur gear pair system," Mechanism and Machine Theory, vol. 107, pp. 229-245, 2017.

[10] J. Y. Tang, S. Y. Chen, and J. Zhong, "A improved nonlinear model for a spur gear pair system," Engineering Mechanics, vol. 25, pp. 217-223, 2008.

[11] Y. X. Wei, J. J. Chen, and H. B. Ma, "Analysis of nonlinear dynamic response of gear-rotor with random parameters," Engineering Mechanics, vol. 29, pp. 319-324, 2012, in Chinese.

[12] A. Kahraman and R. Singh, "Non-linear dynamics of a spur gear pair," Journal of Sound and Vibration, vol. 142, no. 1, pp. 49-75, 1990.

[13] A. Kahraman and R. Singh, "Interactions between timevarying mesh stiffness and clearance non-linearities in a geared system," Journal of Sound and Vibration, vol. 146, no. 1, pp. 135-156, 1991.

[14] T. Sun, Nonlinear Dynamics Research on Planetary Gear Trains, Northwestern Polytechnical University, Xi'an, China, 2000.

[15] Z. M. Sun, Y. W. Shen, and S. Y. Li, "Study on dynamic behavior of encased differential gear train," Chinese Journal of Mechanical Engineering, vol. 38, pp. 44-48, 2002.

[16] W. H. Huang, S. B. Xia, Y. H. Jiao et al., Basic Design Theory and Methods of Nonlinear Dynamics for Rotating Machinery, Science Press, Beijing, China, 2006.

[17] Z. H. Zhang, Numerical Calculation of Powerplant Vibration, Harbin Engineering University Press, Harbin, China, 2007.

[18] M. Maatar and P. Velex, "An analytical expression for the time-varying contact length in perfect cylindrical gears: some possible applications in gear dynamics," Journal of Mechanical Design, vol. 118, no. 4, pp. 586-589, 1996.

[19] R. G. Parker and J. Lin, "Mesh phasing relationships in planetary and epicyclic gears," Journal of Mechanical Design, vol. 126, no. 2, pp. 365-370, 2004. 


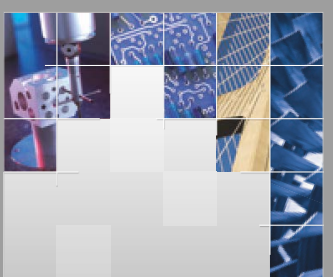

\section{Enfincering}
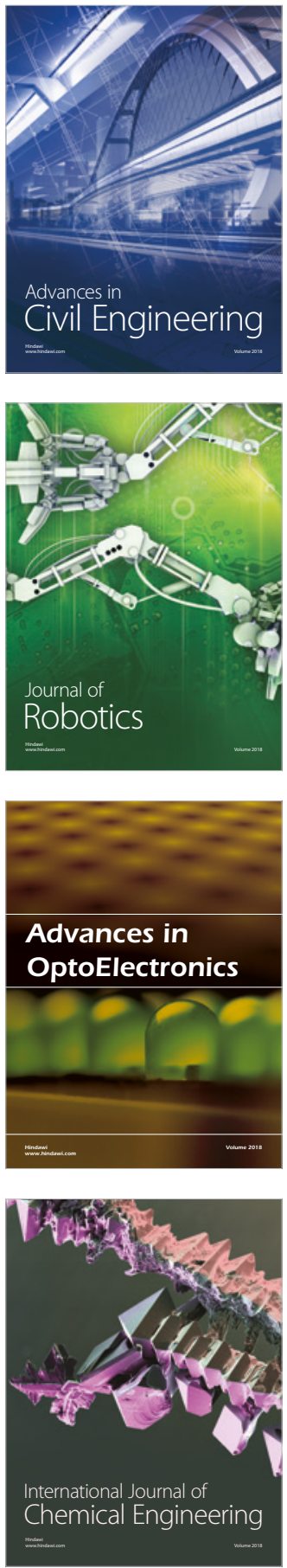

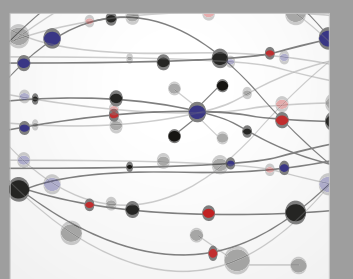

\section{Rotating \\ Machinery}

The Scientific World Journal

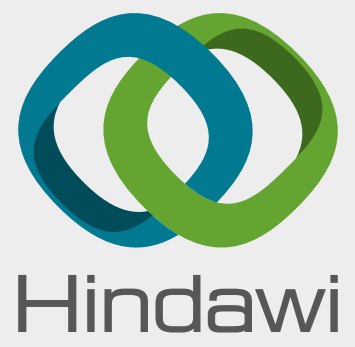

Submit your manuscripts at

www.hindawi.com
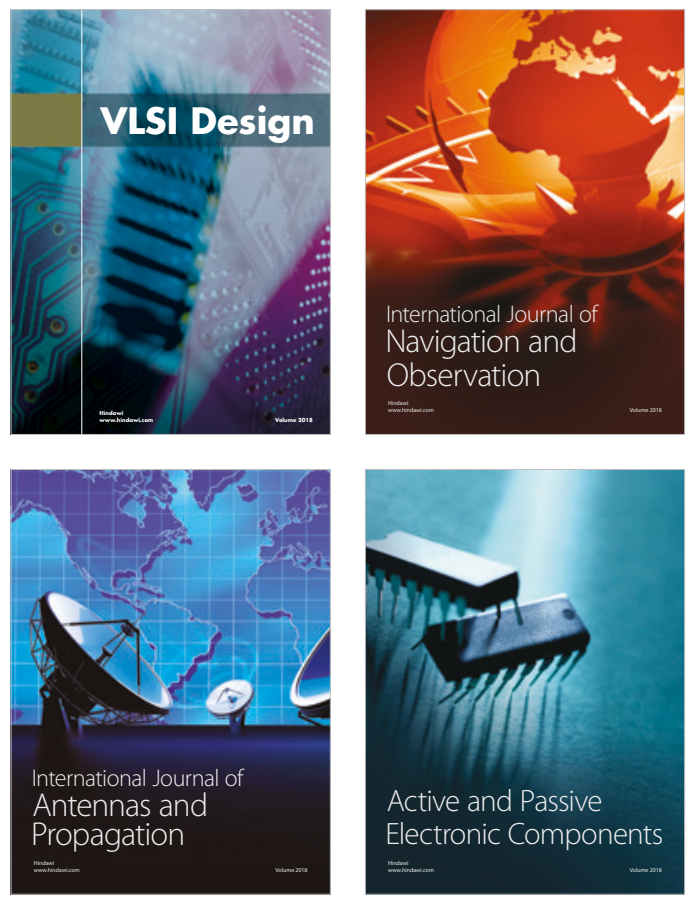
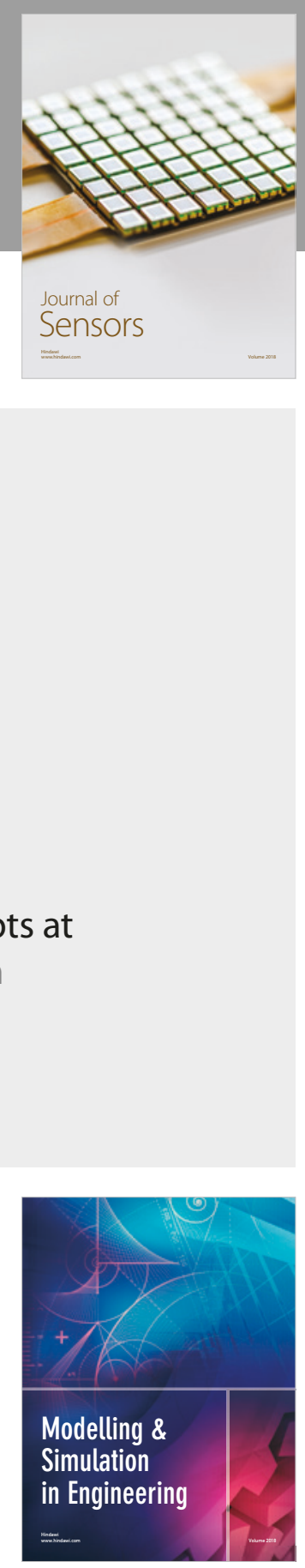

\section{Advances \\ Multimedia}
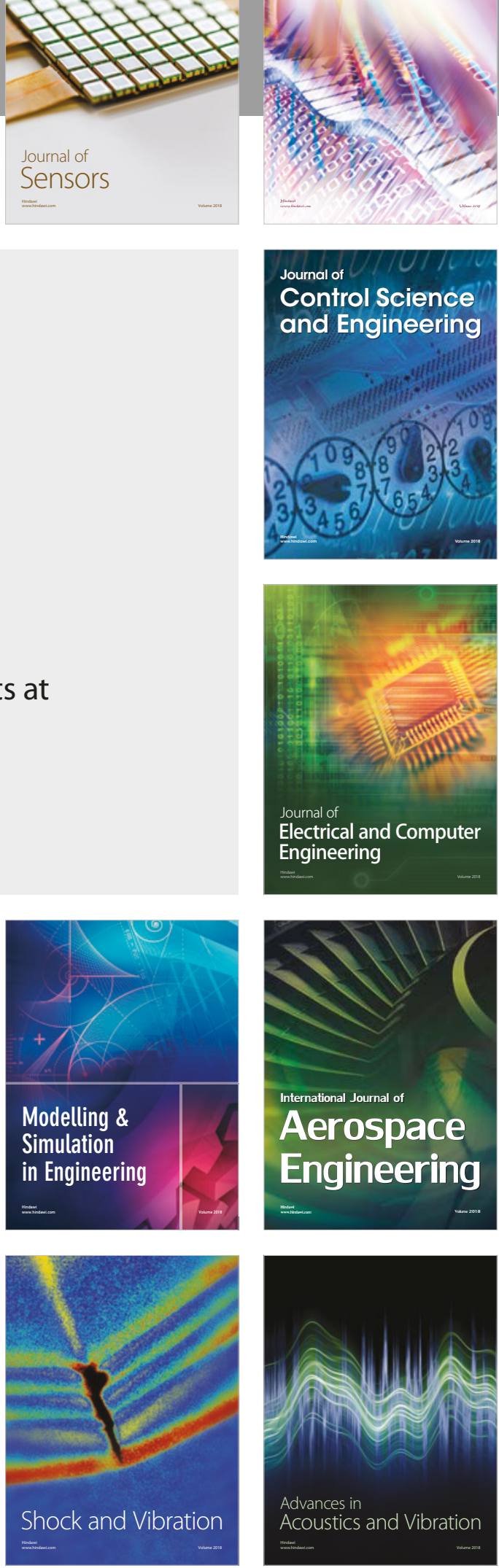